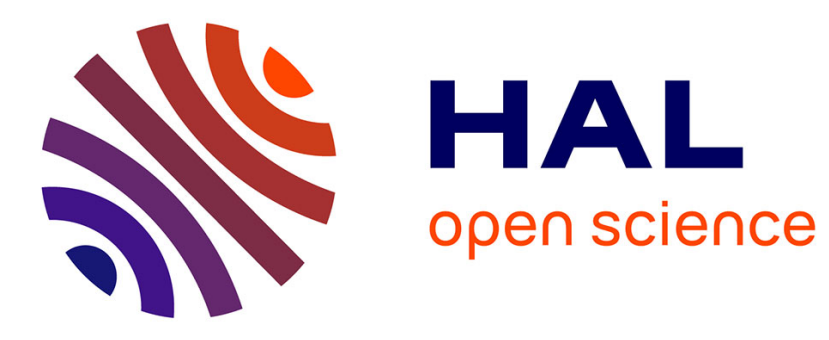

\title{
Pushed global modes in weakly inhomogeneous subcritical flows.
}

\author{
Arnaud Couairon, Jean-Marc Chomaz
}

\section{To cite this version:}

Arnaud Couairon, Jean-Marc Chomaz. Pushed global modes in weakly inhomogeneous subcritical flows.. Physica D: Nonlinear Phenomena, 2001, 158 (1-4), pp.129-150. 10.1016/S0167-2789(01)003268. hal-01025907

\section{HAL Id: hal-01025907 \\ https://hal-polytechnique.archives-ouvertes.fr/hal-01025907}

Submitted on 8 Dec 2017

HAL is a multi-disciplinary open access archive for the deposit and dissemination of scientific research documents, whether they are published or not. The documents may come from teaching and research institutions in France or abroad, or from public or private research centers.
L'archive ouverte pluridisciplinaire HAL, est destinée au dépôt et à la diffusion de documents scientifiques de niveau recherche, publiés ou non, émanant des établissements d'enseignement et de recherche français ou étrangers, des laboratoires publics ou privés. 


\title{
Pushed global modes in weakly inhomogeneous subcritical flows
}

\author{
A. Couairon ${ }^{\mathrm{a}, *}$, J.-M. Chomaz ${ }^{\mathrm{b}}$ \\ a Centre de Physique Théorique (CPhT), CNRS UMR 7644, École Polytechnique, F-91128 Palaiseau, France \\ b Laboratoire d'Hydrodynamique (LadHyX), CNRS UMR 7646, École Polytechnique, F-91128 Palaiseau, France
}

\begin{abstract}
A new type of nonlinear global mode (or fully nonlinear synchronized solution), arising in the dynamics of open shear flows which behave as oscillators [Annu. Rev. Fluid Mech. 22 (1990) 473] and in optical parametric oscillators [J. Opt. Soc. Am. B 17 (2000) 997], is identified in the context of the real subcritical Ginzburg-Landau equation with slowly varying coefficients. The nonlinear global modes satisfy a boundary condition accounting for the inlet of the flow. We show that the spatial structure of these new nonlinear global modes consists of a localized state limited by a pushed upstream front that withstand the mean advection and a fast return to zero downstream achieved either by a second stationary front facing backward, or by a saddle-node bifurcation driven by the non-parallelism of the flow. We derive scaling laws for the slope of the nonlinear global modes at the inlet and for the position of the maximum amplitude which are in agreement with similar scaling obtained in experiments with a shear layer in a Hele-Shaw cell [Phys. Rev. Lett. 82 (1999) 1442]. Extension to the complex Ginzburg-Landau equation is discussed. In a large region of parameters, the nonlinear global modes have the same spatial structure as in the real case and oscillate at a global frequency selected at threshold by the pushed front. In parameters region where these pushed global modes are not selected, new states with a non-periodic in time behavior are exhibited.
\end{abstract}

Keywords: Hydrodynamic stability; Nonlinearity; Pattern selection; Stability of laminar flows

\section{Introduction}

Several spatially developing open shear flows such as bluff body wakes or hot jets are now well known to belong to a particular class of flows which behave like oscillators [1]. The relevant mechanism sustaining the oscillations is likely to rely on the existence of a nonlinear self-sustained synchronized structure, the so-called nonlinear global mode, that represents the distribution of the velocity fluctuations tuned at a specific frequency. This mechanism has received growing attention over the last decade since it does not need any feedback loop to sustain the oscillations. The association of three physical ingredients is sufficient to obtain a nonlinear global mode: (i) there is a linearly unstable basic state; (ii) the geometry is open, i.e., fluid particles enter and leave the experimental domain of interest

\footnotetext{
* Corresponding author.

E-mail address: couairon@cpht.polytechnique.fr (A. Couairon).
} 
and the mean advection must be considered; (iii) nonlinearity saturates the linear instability. A right balance between these ingredients has explained the occurrence of self-sustained synchronized structures in parallel open shear flows including convection cells with throughflow or Taylor-Couette flow with crossflow [4-8]. In the astrophysical field, this approach has been also adopted by Tobias et al. $[9,10]$ to investigate similar structures describing the dynamo wave in spherical geometry. The same ideas have been applied to non-parallel open flows [11-13], and to nonlinear dynamo waves riding on a varying background [14,15]. In all these cases, the existence of a nonlinear global mode has been closely related to a linear transition from convective to absolute instability. In the present paper, we consider systems with stronger nonlinearities and show that a new type of nonlinear global mode arise, that does not rely on such a transition.

The notions of absolute and convective instabilities initially developed in the context of plasmas physics [16-18] constitute the association of the first two ingredients and characterize the impulse response of a system with no streamwise variation (parallel flow). If localized disturbances are growing but swept away from the source, the basic state is said to be convectively unstable. In other words, the mean advection velocity is sufficiently high in comparison with the growth of perturbations to impose that the amplitude returns back to the basic state at a fixed location. By contrast, if linear disturbances spread and grow upstream and downstream and contaminate the whole medium the basic state is said to be absolutely unstable. In the presence of stabilizing nonlinearity, an open flow supports a nonlinear global mode when it is absolutely unstable. In a semi-infinite domain where the origin represents the inlet of the flow, the spatial structure of the global mode is that of a front which withstands the mean advection and is stopped at a finite distance of the inlet (see Fig. 1 and Refs. [19,20]); this distance is called the growth length of the global mode. In this respect, the problem of front selection has advantageously shed light on the existence conditions of nonlinear global modes in homogeneous systems. In an infinite domain, the interface between a stable non-uniform state and an unstable or metastable homogeneous state constitutes a front propagating into the unstable state at a constant velocity in several experiments [21-23]. The determination of the principles governing the selection of the front velocity as well as the pattern behind the front, its oscillation frequency and the front relaxation has been the topic of active experimental and theoretical research since the early paper by Kolmogorov [24-41]. To be concise, the front propagation may be of two types (pulled or pushed regime) schematically depending on whether the state into which the front propagates is unstable or metastable. A linear (pulled regime) and a nonlinear (pushed regime) marginal stability criterion have been proposed to explain the selection [28-32]. The connection between the front selection problem and the notions of absolute and convective instabilities may be made by rephrasing the linear selection principle: the pulled front also referred to as the Kolmogorov front [24,25], is such that, in the frame moving with the front, the basic state is marginally absolutely unstable, i.e., at the threshold between convective and absolute instability. The front is pulled in the sense that its selection among a continuous family, parametrized by its frequency and its velocity, is uniquely determined by the linear properties of the unstable medium in which it propagates. In contrast, the pushed front is a faster front that is, therefore, selected provided it satisfies certain causality conditions [42].

A first type of nonlinear global mode has been shown to exist in semi-infinite domains with a mean advection, when a bifurcation parameter exceeds the absolute instability threshold. These modes, therefore, rely on the selection of a pulled front stopped in its motion by the upstream boundary. The boundary condition at the inlet breaks the Galilean invariance and slightly distorts the front shape and frequency. The case of homogeneous (parallel) flows achieved only in Taylor-Couette rolls or Rayleigh-Bénard convection with throughflow has been solved $[7,8]$ by determining these distortions from the pulled front. Scaling laws previously obtained [4-6] by numerical integration of Navier-Stokes equations for the growth length of the global modes have been found to agree remarkably well with the analytical scaling laws we derived using the complex Ginzburg-Landau equation (see also the related studies in astrophysics $[9,10])$. 
Most open flows, however, cannot be considered parallel. They develop not only in the streamwise direction, but also in the transverse direction and the effect of inhomogeneity (or non-parallelism) has to be explicitly taken into account. In a previous study [11], we have shown that the real supercritical Ginzburg-Landau equation associated with a weakly varying parameter is surprisingly well suited to describe bluff body wakes, which constitute the archetype of self-resonant open shear flows. Indeed, the spatial structure of numerically [43] or experimentally [44] measured wakes has been found to be in accordance with the analytically derived structure of the nonlinear global modes of our model, which still possess the shape of a pulled front, but the saturated part of the solution smoothly returns to zero in the tail. Borrowing the front terminology, these nonlinear global modes will be referred to as "pulled global modes". We have derived scaling laws for the maximum amplitude of the pulled global modes and its position which compare satisfactorily with those found numerically by Zielinska and Wesfreid [43] or experimentally by Goujon-Durand et al. [44]. Similar nonlinear global modes have been identified for optical parametric oscillators $[45,46]$ and in amplitude evolution models of solar and stellar magnetic activity cycles $[14,47]$. There is an extensive literature on these structures in the astrophysical context for which the reader is referred to Ref. [15] and references therein for a review. A locally absolutely unstable region in the flow is necessary to trigger the nonlinear global instability and the emergence of a pulled global mode. The latter scenario shown in Ref. [11] for semi-infinite domains was already valid for the occurrence of a self-sustained linear global mode [48] and is still valid for the emergence of pulled global modes in an infinite domain governed by the supercritical Ginzburg-Landau equation with varying coefficients, thoroughly studied by Pier et al. [12,13].

As expected from the front selection problem, however, a second type of nonlinear global mode exists, the spatial structure of which is associated with an upstream front belonging to the class of pushed fronts and separating the inlet from the saturated amplitude. Still borrowing the front terminology, these modes will be called "pushed global modes". In homogeneous fully nonlinear systems, addition of a generic nonlinearity such as a weak subcritical effect or nonlinear advection promotes the emergence of pushed global modes [19,20,49]. In this case, the medium does not need to be absolutely unstable; nonlinear absolute instability in the sense of Chomaz [50] is sufficient. The recent experiments by Gondret et al. [3] for a Kelvin-Helmholtz sheared interface in a Hele-Shaw cell have shown experimental evidence, for the first time in a fluid system, of a nonlinear transition from convective to absolute instability through a weak subcriticality. In this experiment, the healing length, defined as the distance from the inlet necessary to reach saturation, has been measured and shown to scale logarithmically as a function of the departure from threshold, in agreement with the scaling obtained theoretically in Refs. $[19,20]$.

The objective of the present paper is to describe the pushed global modes in non-parallel open shear flows where nonlinearity is destabilizing. The identification of these self-synchronized structures is now timely. We address the question of their existence and scalings in non-parallel subcritical systems. The rising part of the pushed global modes is steeper than that of the pulled global modes [11], as will be shown by the particular scaling laws for the slope and the position of the maximum amplitude. Moreover, the pushed global modes do not exhibit a smooth tail as the pulled modes obtained in supercritical systems [11]; instead, a second front facing backward is present downstream with amplitude decreasing back to zero. The existence of pushed global modes in optical parametric oscillators has been shown numerically very recently [2]. In open shear flows, these modes are likely to arise in subcritically unstable systems such as Poiseuille or Couette flow in a slow diverging pipe, Görtler flow or wakes confined in a channel. Gondret et al.'s experiment performed in a slowly diverging Hele-Shaw cell could constitute a particular setup suited to test the emergence and scalings of these modes.

The outline of the paper is as follows: In Section 2, we present our real Ginzburg-Landau model which is not rationally derived from fluid equations describing the dynamics of a particular open flow, but is to be seen as an idealized model with the necessary physical ingredients to describe the self-sustained oscillations. In Section 3, we will present qualitatively the spatial structure of the pushed global modes obtained for this model. In Section 4, 
scaling laws for the slope and the position of the maximum amplitude of the nonlinear global modes are explicitly derived. Numerical simulations are presented in Section 5 and show that pushed global modes persists when our model is extended to the case of the complex Ginzburg-Landau equation. Finally, Section 6 is devoted to the discussion of results and conclusion.

\section{The subcritical Ginzburg-Landau model}

We will consider a toy model that possesses all the necessary physical ingredients to mimic the behavior of an open subcritical shear flow such as the Poiseuille flow, with an upstream boundary from which perturbations develop. Moreover, the flow is allowed to evolve slowly in space. The nonlinear self-sustained oscillations, or nonlinear global modes, that such a flow display under certain conditions are usually tuned at a well-defined frequency. In the present paper, we will first focus on the spatial structure of these modes. We will then briefly go back to the frequency selection problem in Section 5 where we will show how the results extend in the oscillatory case. Our toy model is, therefore, constituted by the real subcritical Ginzburg-Landau equation with a positive advection velocity $U_{0}$ which models the mean flow, and a slowly varying coefficient $\mu(x)$ :

$$
\frac{\partial A}{\partial t}+U_{0} \frac{\partial A}{\partial x}=\frac{\partial^{2} A}{\partial x^{2}}+\mu(x) A+A^{3}-A^{5} .
$$

We will present the spatial structure of nonlinear global (NG) modes which are steady solutions of (1) in a semi-infinite domain representing the region in which the flow develops, and satisfying the ideal inlet condition

$$
A(x=0)=0,
$$

accounting for a zero level perturbation. The second boundary condition is the asymptotic behavior at $x=+\infty$ dictated by the fact that the system is assumed here to be sufficiently stable at infinity. We, therefore, consider only solutions asymptotic to 0 when $x=+\infty$ (i.e., such that $\mu(+\infty)<-\frac{1}{4}$ ).

$$
A(+\infty)=0 .
$$

The latter condition may easily be relaxed: for example if the bifurcation parameter is asymptotic to a constant at infinity $(\mu(+\infty)=\mathrm{Cst})$, this condition would be that $A$ reach at infinity a minimum of the potential from which terms of the right-hand side of Eq. (1) derive (i.e., $V(A)=\frac{1}{2} \mu A^{2}+\frac{1}{4} A^{4}-\frac{1}{6} A^{6}$ ). In the same manner, only the local bifurcation parameter $\mu(x)$ varies in space in our model because this assumption is sufficient to obtain the spatial structure of NG modes; however our analysis applies when each coefficient in front of the terms of Eq. (1) varies.

In order to give a concrete example, the bifurcation parameter is assumed to depend linearly on the space variable $x$

$$
\mu(x)=\mu_{0}-\mu_{1} x
$$

where $\mu_{0}$ and $\mu_{1}$ are positive constants. Therefore, the system is similar to the one used in $[11,48]$ but with a subcritical nonlinear potential. The choice of a linear dependence in Eq. (4) does not restrict the generality of the study which remains valid as long as $\mu(x)$ is any slowly decreasing function depending on $x$ only through a slow space variable $X=x / L$ ( $L$ measures the inhomogeneity length scale and is equal to $\mu_{1}^{-1}$ in the present case). Since the spatial structure of the NG modes strongly relies on the results found in the constant $\mu$ case, we begin with a brief summary of the latter results. 


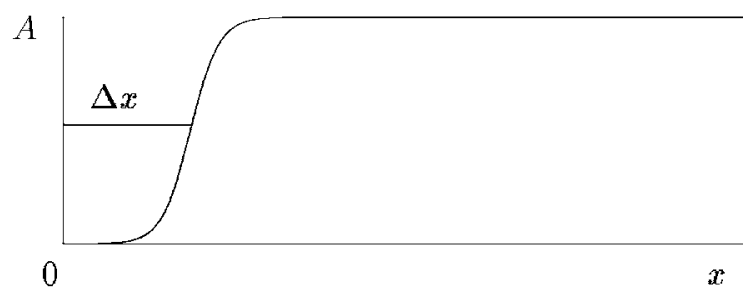

Fig. 1. Spatial structure of a homogeneous nonlinear global mode.

\subsection{Constant $\mu$ case}

The constant $\mu$ case models parallel open flows, i.e., flows where the velocity profile constituting the basic state does not vary in the direction transverse to the flow. In the parallel flow case $\left(\mu_{1}=0\right.$ and $\left.\mu=\mu_{0}\right)$, we have determined in [20], for the same subcritical Ginzburg-Landau model, the transition to a global instability and the associated spatial structure of steady solutions of Eq. (1) vanishing at the origin and saturating at a finite amplitude when $x \rightarrow+\infty$. When the bifurcation parameter does not vary with respect to $x$, in order to avoid confusion, these solutions will be denoted "homogeneous" nonlinear global (HNG) modes throughout the study. In this case, an NG mode satisfies at $x=0$ the boundary condition (2) and at infinity, Eq. (3) is replaced by $A(+\infty)=A_{2}\left(\mu_{0}\right)$ with

$$
A_{2}(\mu)=\left(\frac{1}{2}+\sqrt{\mu+\frac{1}{4}}\right)^{1 / 2}
$$

Fig. 1 shows the spatial structure of such an HNG mode constituted by a front halted in its upstream motion by the boundary condition and separating the inlet at $x=0$ from the saturated region.

HNG modes exist in the shaded region (Fig. 2) of parameter space $\left(U_{0}, \mu\right)$ limited by $\mu>\mu_{A}\left(U_{0}\right)$ with

$$
\begin{aligned}
& \mu_{A}\left(U_{0}\right)=\frac{3}{16} U_{0}^{2}+\frac{1}{8} \sqrt{3} U_{0}-\frac{3}{16} \text { if } U_{0}<\sqrt{3}, \\
& \mu_{A}\left(U_{0}\right)=\frac{1}{4} U_{0}^{2} \quad \text { if } U_{0}>\sqrt{3} .
\end{aligned}
$$

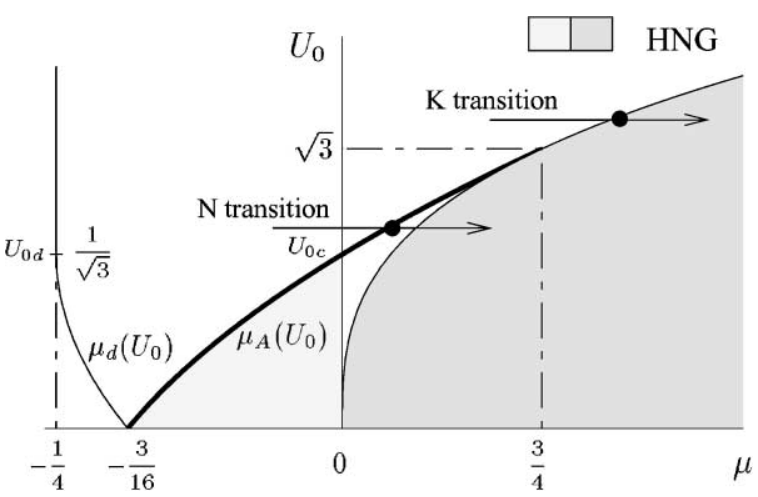

Fig. 2. Parameter space of the homogeneous problem. For the sake of clarity, the axis scales are not linear. HNG modes exist in the shaded regions. For $U_{0}>\sqrt{3}$, the transition to global instability is of the Kolmogorov (K) type (simultaneous with the transition from convective to absolute instability). For $U_{0}<\sqrt{3}$ the transition is of nonlinear type $(\mathrm{N})$, occurring while the basic state is convectively unstable or stable. The fine curve illustrates the $\mathrm{K}$ transition and bounds the (dark gray) region of absolute instability. The curve $\mu_{\mathrm{d}}\left(U_{0}\right)$ and the quantities $U_{0 \mathrm{~d}}$ and $U_{0 \mathrm{c}}$ are discussed in the text. 
In [20], depending on whether (6) or (7) holds, we have characterized the conditions of emergence of these HNG modes and we have determined two different scaling laws for their growth length $\Delta x$, versus the departure from global instability threshold

$$
\epsilon=\mu-\mu_{A}
$$

The quantity $\Delta x$ is defined as the distance at which the solution reaches $50 \%$ of its maximum amplitude $A_{2}$.

- When $U_{0}>\sqrt{3}$, the threshold for the existence of a global mode does correspond to the change in the linear instability of the basic solution $A=0$ from convective to absolute. HNG modes exist only when small amplitude waves are absolutely unstable (dark gray region of Fig. 2 limited by $\mu=\frac{1}{4} U_{0}^{2}$ ). In this case, the transition has been called of Kolmogorov type since it corresponds to a Kolmogorov front [24] blocked on the origin. The growth length $\Delta x$ scales as

$$
\Delta x=\frac{\beta}{\sqrt{\epsilon}} \quad(\text { Kolmogorov K type })
$$

where $\beta=\pi$ for Eq. (1). A similar scaling in $\epsilon^{-1 / 2}$ has been found for the growth length of HNG modes obtained for the supercritical real or complex Ginzburg-Landau model [19,20], for which the transition to global instability is always of the Kolmogorov type. Although the quantity $\epsilon^{-1 / 2}$ is the natural Ginzburg-Landau length scale, the coefficient $\beta$ is obtained exactly by non-trivial matched asymptotic expansions. This scaling has been experimentally and numerically verified for Rayleigh-Bénard convection with throughflow and Taylor-Couette experiments with throughflow $[7,8]$.

- When $U_{0}<\sqrt{3}$, the threshold of existence (6) of an HNG mode is not linked to a change in the nature of the linear wave instability, but from nonlinear effects that are able to withstand the advection. Therefore, the global modes obtained in that case have been called of nonlinear type (following a classical differentiation made for front solutions [25]). HNG modes exist while the system is convectively unstable or stable (light gray region of Fig. 2). For fixed $U_{0}$, when increasing $\mu$, the global instability occurs before the absolute/convective transition (it is, therefore, called "nonlinear transition") and the HNG modes are much steeper than in the previous case with a growth length scaling as $\log (1 / \epsilon)$ :

$$
\Delta x \simeq \log \left(\frac{1}{\epsilon}\right) \quad \text { (Nonlinear N type) }
$$

This scaling has very recently been observed for a Kelvin-Helmholtz sheared interface in a Hele-Shaw cell [3].

\subsection{Varying $\mu$ case}

In the varying $\mu$ case ( $\mu_{1} \neq 0$ or non-parallel flow case), three different types of NG modes may be predicted whose spatial structure is displayed schematically in Fig. 3. Although these structures have been obtained for the specific model that we present below, they are generic and may appear in any nonlinear system exhibiting synchronized structures.

At the origin of the semi-infinite domain, we assume that the bifurcation parameter $\mu_{0}$ is larger than the NG instability threshold, i.e., the threshold $\mu_{A}\left(U_{0}\right)$ (Eq. (6)) of emergence of HNG modes:

$$
\mu_{0}=\mu_{A}+\epsilon
$$

If $\epsilon$ is sufficiently large, an NG mode may grow in space and saturate before the local bifurcation parameter $\mu(x)$ has become smaller than $\mu_{A}\left(U_{0}\right)$, whose dependence in $U_{0}$ will no longer be mentioned for the sake of clarity. 

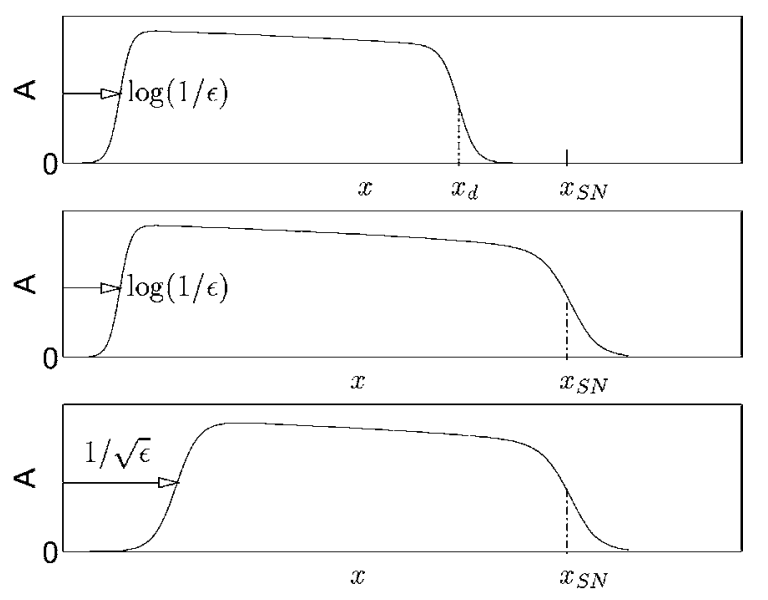

Fig. 3. Schematic spatial structure of the different types of nonlinear global modes predicted by the present model. (a) $U_{0}<U_{0 \mathrm{~d}} \equiv 1 / \sqrt{3}$ : a pushed global mode made of an upstream pushed front and ended by a backward facing front. (b) $U_{0 \mathrm{~d}}<U_{0}<\sqrt{3}$ : a pushed global mode with a tail returning to zero through a saddle-node bifurcation. (c) $U_{0}>\sqrt{3}$ : a pulled global mode with the same type of tail as in (b).

Therefore, we will not only use a weakly non-parallel hypothesis $\left(\mu_{1} \ll 1\right)$, but also a stronger condition ensuring that nonlinearity "dominates" over non-parallelism in a sense that we will precise. As in [11], we face here a singular perturbation problem as the two limits $\mu_{1} \rightarrow 0$ and $\epsilon \rightarrow 0$ cannot be taken at the same time and respective orders have to be specified (non-uniform limit). The physical guideline for the ordering in small parameters relies on comparing the typical length scales associated with nonlinearity (departure from threshold $\epsilon$ ) quantified by $\Delta x$ and the length scale of the inhomogeneity characterized by the distance $x_{A}$ from the origin at which $\mu\left(x_{A}\right)=\mu_{A}$ $\left(x_{A}=\epsilon / \mu_{1}\right.$ in this study). If the domain were not limited at $x=0$ but were extended toward $x=-\infty$, a front located at $x<x_{A}$ would be moving upstream, whereas a front located at $x>x_{A}$ would be moving downstream. If the growth length $\Delta x$ of HNG modes is large compared to the inhomogeneity length scale $x_{A}$, the front would reach saturation in a domain where it cannot sustain the mean advection and the system would relax to zero. When $\Delta x$ is sufficiently small in comparison with $x_{A}\left(\Delta x \ll x_{A}\right)$, the front saturates in a region where it is able to withstand the advection and a self-sustained mode (NG mode) may exist. We can describe the spatial structure of this NG mode, in this case a steady solution of Eq. (1), i.e., one of the solutions of the equation

$$
\frac{\mathrm{d}^{2} A}{\mathrm{~d} x^{2}}-U_{0} \frac{\mathrm{d} A}{\mathrm{~d} x}+\left(\mu_{0}-\mu_{1} x\right) A+A^{3}-A^{5}=0,
$$

vanishing at $x=0$ and $x \rightarrow+\infty$, by interpreting its growing part as the leading edge of an HNG mode (with the constant parameters $\mu=\mu_{0}$ and $\left.U_{0}\right)$ and its decreasing part as the adiabatic variation of the saturation amplitude $A_{2}(\mu)$ with respect to $x$ through $\mu(x)$. Let us also introduce $x_{\mathrm{s}}$, the position of the maximum amplitude of the NG mode.

In the case $U_{0}<\sqrt{3}$ (Fig. 3(a) and (b)), a nonlinear transition occurs in the corresponding homogeneous problem and the NG mode is, therefore, a pushed global mode, i.e., its upstream front is close to a pushed front. Its maximum amplitude is reached at $x_{\mathrm{s}} \sim \Delta x \sim \log (1 / \epsilon)$. Using formula (10) for the growth length of HNG modes, the condition that $\Delta x \ll x_{A}$ gives

$$
\Delta x \ll \frac{\epsilon}{\mu_{1}} \Rightarrow \mu_{1} \ll \frac{\epsilon}{\log (1 / \epsilon)} .
$$

At this stage, we must anticipate on the result which will be found for $x_{\mathrm{s}}$ in order to set correctly the condition of validity of the analysis, which turns out to be slightly different from (13): $x_{\mathrm{s}}$ will be found to possess a dominant 
contribution which scales as $\log \left(1 / \mu_{1}\right)$, and therefore exceeds the expected contribution $\log (1 / \epsilon)$ coming from $\Delta x$ (as if the NG mode was homogeneous). The condition $x_{\mathrm{s}} \ll x_{A}$ is more restrictive than $\Delta x \ll x_{A}$ and then reads

$$
\frac{\epsilon}{\mu_{1}} \gg \log \left(\frac{1}{\mu_{1}}\right) \text {. }
$$

Two situations may occur for the tail of this mode. In both cases, the tail is slave of the upstream structure of the solution.

When $U_{0}<U_{0 \mathrm{~d}} \equiv 1 / \sqrt{3}$ (Fig. 3(a)), the NG mode then follows adiabatically $A_{2}(\mu(x))$ and decreases to zero at $x_{\mathrm{d}}$, where $A_{2}\left(\mu\left(x_{\mathrm{d}}\right)\right)$ is such that a backward facing front linking $A_{2}$ to zero exists. Such a front exists for the parameters belonging to the fine line for $-\frac{1}{4}<\mu<-\frac{3}{16}$ in Fig. 2.

When $U_{0 \mathrm{~d}}<U_{0}<\sqrt{3}$ (Fig. 3(b)), the NG mode follows adiabatically $A_{2}(\mu(x))$ till the solution disappears in a saddle-node bifurcation at $x_{\mathrm{SN}}$, for which $\mu\left(x_{\mathrm{SN}}\right)=-\frac{1}{4}$.

In the case $U_{0}>\sqrt{3}$ (Fig. 3(c)), the Kolmogorov transition occurs in the corresponding homogeneous problem; the structure of the NG mode is nearly identical to that obtained for the supercritical model presented in [11]. The NG mode is not a pushed global mode but a pulled global mode since its upstream front is close to a pulled front. The results in this case constitute a straightforward extension of the case described in [11] and will not be fully discussed here to avoid redundancies. The maximum amplitude of the pulled mode scales as $x_{\mathrm{s}} \sim 1 / \sqrt{\epsilon}$ and the condition that $x_{\mathrm{S}} \ll x_{A}$ gives the validity range where these modes may be found ( $\epsilon \gg \mu_{1}^{2 / 3}$ in this model). Only the downstream part of the structure is different: instead of a smooth return to zero, it is constituted by a sharp return to zero though a saddle-node bifurcation at $x_{\mathrm{SN}}$ such that $\mu\left(x_{\mathrm{SN}}\right)=-\frac{1}{4}$, similar to that obtained for $U_{0 \mathrm{~d}}<U_{0}<\sqrt{3}$.

We will, therefore, restrict our presentation to the range $0 \leq U_{0}<\sqrt{3}$ which concerns pushed global modes. Our goal is to describe the spatial structure of pushed global modes when condition (14) is satisfied. In particular, we seek a scaling law for the position $x_{\mathrm{s}}$ of the maximum amplitude. Let us emphasize that $x_{\mathrm{s}}$ is expected to be at least greater than the growth length of HNG modes $\log (1 / \epsilon)$.

\section{Spatial structure of pushed global modes}

A generic example of the ultimate state, i.e., a pushed global mode obtained in a temporal numerical simulation of Eq. (1), with vanishing boundary conditions at the origin and at infinity, is displayed in Fig. 4. The advection velocity was here smaller than $U_{0 \mathrm{c}} \equiv 1 / \sqrt{3}$ (see Fig. 2), the front velocity of a pushed front propagating in a neutrally stable medium. The bifurcation parameter $\mu_{0}$ was negative and the medium was then stable near the origin. Initially, the system was set in a localized finite amplitude state. After destabilization, the system eventually converged to the steady state whose spatial structure is shown in Fig. 4 . When the advection velocity is in the range $[1 / \sqrt{3}, \sqrt{3}]$, a steady state such as that displayed in Fig. 4 is obtained asymptotically for any initial condition of finite or vanishing amplitude. Eqs. (4), (6) and (11) indeed show that $\mu_{A}\left(U_{0}\right)>\mu_{A}\left(U_{0 \mathrm{c}}\right)=0$ and $\mu(x)>0$ near the origin. Thus the medium is linearly unstable in a region of finite extent beyond $x=0$ and an NG mode is triggered by the growth of infinitely small perturbations from the uniform initial state $A=0$. Only the tail differs in the latter case, since the return to zero is given by a saddle-node bifurcation. Note that the fact that $U_{0 \mathrm{c}}=U_{0 \mathrm{~d}}$ is a particular case pertaining to the real Ginzburg-Landau model but in general, $U_{0 \mathrm{c}} \neq U_{0 \mathrm{~d}}$.

The spatial structure of the pushed global mode will be described in the general case by using the method of matched asymptotic expansions [51]. We distinguish seven subdomains in the original semi-infinite domain represented in Fig. 4.

The nonlinear front layers $\mathrm{NF}^{\mathrm{s}}$ (saturated), $\mathrm{NF}^{\mathrm{d}}$ (downstream), the central nonlinear layer $\mathrm{CNL}$ and a linear outer layer $\mathrm{OL}$ are separated by transition layers (inner layers), namely, $\mathrm{IL}$ at the origin, $\mathrm{TL}^{\mathrm{s}}$ around $x_{\mathrm{S}}$ the location of the 


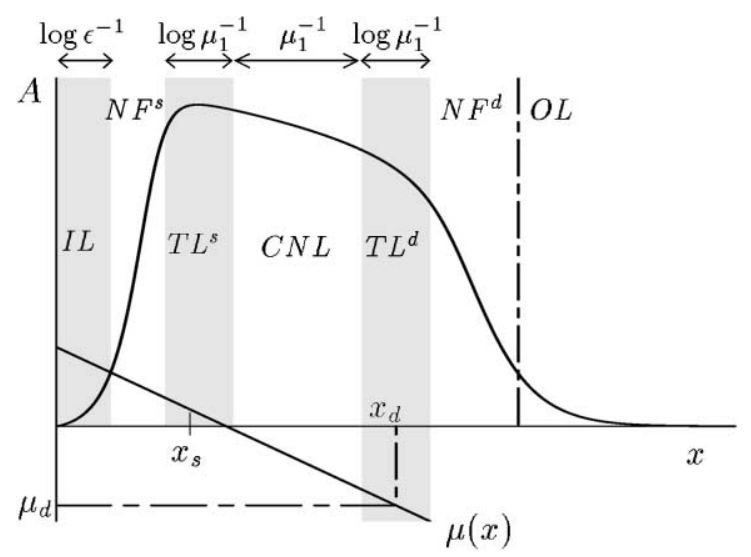

Fig. 4. An NG mode displays clearly four parts: in the nonlinear front layer $\mathrm{NF}^{\mathrm{s}}\left(\mathrm{NF}^{\mathrm{d}}\right)$, the solution steepens while growing (decreasing). In the central nonlinear layer (CNL), the solution decreases softly. OL is an outer linear region where the amplitude is small. An inner layer (IL) at the origin, and two transition layers $\left(\mathrm{TL}^{\mathrm{s}}\right.$ around the maximum amplitude, $\mathrm{TL}^{\mathrm{d}}$ around the end of the weakly varying part of the solution) connect linear and nonlinear regions. The solid line represents the bifurcation parameter $\mu(x)$.

maximum amplitude, $\mathrm{TL}^{\mathrm{d}}$ around the point $x_{\mathrm{d}}$ which represents the right boundary of CNL and will be specified below. The respective sizes of the layers are indicated in Fig. 4. In the following, we indicate only briefly the nature of the solutions in each layers. The size of the different layers may be obtained only by the matching, the mathematical details of which are postponed in Appendix A.

\subsection{Qualitative description of pushed global modes}

In each subdomain, we must solve Eq. (12) and we need two boundary conditions in order to find the corresponding solution. Since we only know the boundary conditions at the origin and at infinity of the whole domain, the matching between the different layers we have introduced will determine all integration constants.

1. Inner layer IL of size $\mathcal{O}\left(\log \epsilon^{-1}\right)$ : since the amplitude remains small, we use a solution of Eq. (12) linearized around zero, and the two integration constants are fixed by the boundary condition at the origin and the matching, detailed below, with the solution in $\mathrm{NF}^{\mathrm{s}}$.

2. Nonlinear front layer $\mathrm{NF}^{\mathrm{s}}$ of size $\mathcal{O}(1)$ : the solution is similar to the homogeneous global mode which grows in space till it reaches its maximum amplitude (Fig. 1) with an added small perturbation due to inhomogeneity. Since this solution is growing on an order one length scale, the bifurcation parameter can be considered as a constant (which equals $\mu_{A}\left(U_{0}\right)$, Eq. (6)) at leading order. We use the study of the parallel model equation (12) with $\mu_{0}=\mu_{A}\left(U_{0}\right)$ to find the corresponding solution and compute the correction due to inhomogeneity.

At $x_{\mathrm{s}}$, the bifurcation parameter is close to the value $\mu_{A}\left(U_{0}\right)$ when neglecting $\epsilon$ and $\mu_{1} \log \epsilon$ terms. The matching between $\mathrm{NF}^{\mathrm{s}}$ and $\mathrm{TL}^{\mathrm{s}}$ is used as a boundary condition for the solution in $\mathrm{NF}^{\mathrm{s}}$. For this reason, the solution in $\mathrm{NF}^{\mathrm{s}}$ can be computed unambiguously, only after the solution in $\mathrm{TL}^{\mathrm{s}}$ is worked out.

3. Transition layer $\mathrm{TL}_{\mathrm{s}}$ of size $\mathcal{O}\left(\log \mu_{1}^{-1}\right)$ : the amplitude has to match with the solution in $\mathrm{NF}^{\mathrm{s}}$ and therefore is close to $A_{2}\left(\mu_{A}\right)$ (Eq. (5)). It is represented by a solution of Eq. (12) linearized around $A_{2}\left(\mu_{A}\right)$. The matching with CNL on the one hand, and the condition that $A(x)$ realizes a maximum at $x_{\mathrm{s}}$ on the other hand determine the two integration constants. Again, the solution in $\mathrm{TL}^{\mathrm{s}}$ can be only determined after the solution in $\mathrm{CNL}$, the next layer downstream, is known. 
4. Central nonlinear layer $(\mathrm{CNL})$ of size $\mathcal{O}\left(\mu_{1}^{-1}\right)$ : it denotes the subdomain where the solution has bifurcated to the finite amplitude state $A_{2}(\mu)$ (Eq. (5)) and follows adiabatically the weak variation of the bifurcation parameter $\mu(x)$. The pushed global mode amplitude decreases slowly from $A_{2}\left[\mu\left(x_{\mathrm{s}}\right)\right]$ to $A_{2}\left[\mu\left(x_{\mathrm{d}}\right)\right]$, following approximately the slowly varying function $A_{2}[\mu(x)]=\left[\frac{1}{2}+\left(\mu_{0}-\mu_{1} x+\frac{1}{4}\right)^{1 / 2}\right]^{1 / 2}$ and reflecting the interplay between nonlinearities and inhomogeneity. $C N L$ boundaries $x_{\mathrm{s}}$ and $x_{\mathrm{d}}$ actually determine the locations where the slope of the solution starts to steepen (with growing or decreasing amplitude). The left boundary $x_{\mathrm{s}}$ has been already defined as the position of the maximum amplitude, but the right boundary $x_{\mathrm{d}}$ which is the position of the decreasing front will be specified below.

5. Transition layer $\mathrm{TL}^{\mathrm{d}}$ of size $\mathcal{O}\left(\log \mu_{1}^{-1}\right)$ around $x_{\mathrm{d}}$ : it plays the same role as $\mathrm{TL}^{\mathrm{s}}$ with $A_{2}\left(\mu_{A}\right)$ replaced by $A_{\mathrm{d}}=A_{2}\left(\mu_{\mathrm{d}}\right), \mu_{\mathrm{d}}=\mu\left(x_{\mathrm{d}}\right)$ and the condition that $A(x)$ be maximum at $x_{\mathrm{s}}$ is replaced by the condition that $A(x)$ exactly be equal to $A_{\mathrm{d}}$ at $x_{\mathrm{d}}$.

6. Nonlinear front layer $\mathrm{NF}^{\mathrm{d}}$ of size $\mathcal{O}(1)$ : the solution decreases back to a small amplitude on an order one length scale and we again consider that this decreasing solution is a stationary backward facing nonlinear front with corrections due to inhomogeneity.

The position $x_{\mathrm{d}}$ of the decreasing front linking the finite amplitude state to zero is determined by considering the existence of the front in the homogeneous problem where the bifurcated state relaxes back to zero. For $U_{0}>U_{0 \mathrm{~d}}\left(U_{0 \mathrm{~d}} \equiv 1 / \sqrt{3}\right.$, see Fig. 2$)$, this occurs through a saddle-node bifurcation, i.e., the saturated state $A_{2}(\mu(x))$ loses its existence at a finite amplitude when $\mu$ decreases and reaches the value

$$
\mu_{\mathrm{d}}\left(U_{0}\right)=-\frac{1}{4} \text { if } U_{0}>\frac{1}{\sqrt{3}} .
$$

For $U_{0}<U_{0 \mathrm{~d}}$, this occurs when the bifurcation parameter reaches a larger value $\mu_{\mathrm{d}}\left(U_{0}\right)$ such that an heteroclinic orbit (which represents the steady front solution in the phase space $(A, \mathrm{~d} A / \mathrm{d} x))$ connects $\left(A_{2}\left(\mu_{\mathrm{d}}\right), 0\right)$ to the origin $(0,0)$. In other words, for $\mu=\mu_{\mathrm{d}}\left(U_{0}\right)$, the stable manifold of the origin of phase space intersects the unstable manifold of the fixed point $\left(A_{2}\left(\mu_{\mathrm{d}}\right), 0\right)$. In a previous study [20], we have determined the decreasing front transition value $\mu=\mu_{\mathrm{d}}\left(U_{0}\right)$ which is plotted in Fig. 2 with a fine line and has parametric equation

$$
\mu_{\mathrm{d}}\left(U_{0}\right)=\frac{3}{16} U_{0}^{2}-\frac{\sqrt{3}}{8} U_{0}-\frac{3}{16} \text { if } U_{0}<\frac{1}{\sqrt{3}} .
$$

Returning to the inhomogeneous problem, the position of the decreasing front is determined by $\mu\left(x_{\mathrm{d}}\right)=\mu_{\mathrm{d}}$.

Let us point out that the whole structure of the pushed global mode has been pinned by the position of CNL between $x_{\mathrm{S}}$ and $x_{\mathrm{d}}$. Actually, Fig. 4 may be viewed as the projection in the $(A, x)$ plane of the three-dimensional phase space $(A, \mathrm{~d} A / \mathrm{d} x, x)$. Fig. 5 shows the structure of the solution in the phase space: the growing and decreasing fronts in $\mathrm{NF}^{\mathrm{s}}$ and $\mathrm{NF}^{\mathrm{d}}$ lay nearly in planes parallel to $(A, \mathrm{~d} A / \mathrm{d} x)$ and correspond to rapid variations of $A$ with $\mu(x)$ nearly frozen, whereas the solution in CNL follows adiabatically the variation of $\mu(x)$ and lays nearly in the $(A, x)$ plane. From the left and right boundaries $x_{\mathrm{s}}$ and $x_{\mathrm{d}}$ of the central nonlinear layer CNL, now precisely known from the variation of $\mu(x)$, we obtain that the size of CNL is $\mathcal{O}\left(\mu_{1}^{-1}\right)$, since $x_{\mathrm{d}} \sim$ $\mu_{1}^{-1}\left[\mu_{A}\left(U_{0}\right)-\mu_{\mathrm{d}}\left(U_{0}\right)\right]$ and $x_{\mathrm{S}} \ll \mu_{1}^{-1}$. The whole solution is, therefore, pinned by CNL since the matching can be performed from $\mathrm{CNL}$ to the nonlinear front layers: solutions in $\mathrm{NF}^{\mathrm{s}}$ and $\mathrm{NF}^{\mathrm{d}}$ can be computed by applying only one boundary condition given by the matching with $C N L$ via $\mathrm{TL}^{\mathrm{s}}$ and $\mathrm{TL}^{\mathrm{d}}$. However, the whole matching must be done in order to verify that the boundary conditions (2) and (3) can be indeed satisfied and to know precisely the restrictions on $\mu_{1}$ and $\epsilon$ these boundary conditions imply. Nevertheless, this constitutes the last stage of the matching and does not alter the spatial structure of the solution described above.

7. Outer layer OL: the solution must vanish at infinity and hence, the amplitude remains small. We, therefore, use a solution of Eq. (12) linearized around zero, and fix the remaining integration constant and the order of magnitude 


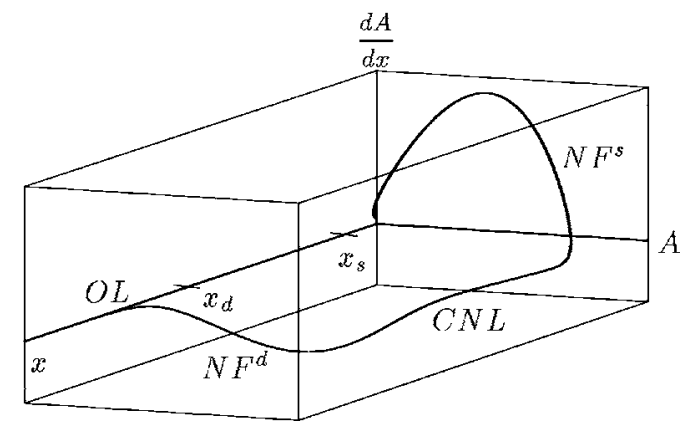

Fig. 5. The solution shown in Fig. 4 is plotted in the phase space $(A, x, \mathrm{~d} A / \mathrm{d} x)$. The solution in $\mathrm{NF}^{\mathrm{s}}$ and $\mathrm{NF}^{\mathrm{d}}$ lay nearly in planes $(A, \mathrm{~d} A / \mathrm{d} x)$, whereas in CNL, it lays nearly in the plane $(A, x)$.

of the amplitude in OL by the matching with $\mathrm{NF}^{\mathrm{d}}$. The matching of the trail in OL is always possible and does not require an extra transition layer since the trail is slaved to the upstream structure of the NG mode.

The complete spatial structure of NG modes represented in Fig. 4 for model (12) is detailed for each layer in Appendix A. Only the matching between the inner layer IL and the front layer $\mathrm{NF}^{\mathrm{s}}$ is detailed in Section 4 since it determines the scaling law for the position $x_{\mathrm{S}}$ of the maximum amplitude of the NG mode.

\section{Matching $\mathrm{IL} \rightarrow \mathrm{NF}^{\mathrm{s}}$ and scaling law for the position of the maximum}

The inner solution in IL and the outer solution in $\mathrm{NF}^{\mathrm{s}}$ are obtained in Appendix A. Denoting by $\xi$ the inner variable for the amplitude in IL, the matching between these layers is done in a plane ( $A, \mathrm{~d} A / \mathrm{d} x)$ (Fig. 5), i.e., we match $\mathrm{d} A / \mathrm{d} x$ (considered as a function of $A$ ) when $A \rightarrow 0$, with $\mathrm{d} \xi / \mathrm{d} x$ when $x \rightarrow+\infty$. When $x \rightarrow+\infty$, the asymptotic behavior of the inner solution (A.32), i.e., $\mathrm{d} \xi / \mathrm{d} x$ as a function of $\xi$ reads as

$$
\frac{\mathrm{d} \xi}{\mathrm{d} x} \simeq r^{+} \xi+v_{0}^{1-r^{-} / r^{+}}\left(r^{+}-r^{-}\right)^{r^{-} / r^{+}-1} \xi^{r^{-} / r^{+}},
$$

where $r^{+}=\frac{1}{4}\left(U_{0}+\sqrt{3}\right)$ and $r^{-}=\frac{1}{4}\left(3 U_{0}-\sqrt{3}\right)$. The quantities $r^{+}$and $r^{-}$represent the spatial exponential growth (or decay) rates linked to possible ways to depart from (or arrive to) zero. The quantity $v_{0}$ represents the slope at the origin of the inner solution (and will be given by the matching). Multiplication of Eq. (17) by $\theta(\epsilon)$ (where $\theta(\epsilon) \rightarrow 0$ is the size in amplitude of the inner layer and will be specified below) and introduction of $A=\theta(\epsilon) \xi$ in Eq. (17) yields the following expansion (inner solution rewritten in outer variable)

$$
\theta(\epsilon) \frac{\mathrm{d} \xi}{\mathrm{d} x} \simeq \frac{A_{\mathrm{s}}^{2}}{\sqrt{3}} A+\left[v_{0} \theta(\epsilon)\right]^{1-\lambda_{1}}\left(r^{+}-r^{-}\right)^{\lambda_{1}} A^{\lambda_{1}},
$$

where $A_{\mathrm{S}} \equiv A_{2}\left(\mu_{A}\right)$ and $\lambda_{1}=r^{-} / r^{+}$. The asymptotic behavior of the solution $u(A) \equiv \mathrm{d} A / \mathrm{d} x(A)$ in $\mathrm{NF}^{\mathrm{s}}$ when $A \rightarrow 0$ is given by Eq. (A.30) of Appendix A. For the matching, we note that $r^{+}=A_{\mathrm{s}}^{2} / \sqrt{3}$. Eq. (18) must be compared with expansion (A.30) in $\mathrm{NF}^{\mathrm{S}}$. The zeroth order terms are identical. At next order, we must identify $\theta(\epsilon)^{1-\lambda_{1}}$ with either $\epsilon$ or $\mu_{1} \log \left(1 / \mu_{1}\right)$. When $\mu_{1} \log \left(1 / \mu_{1}\right) \ll \epsilon, \epsilon$ is the dominant term in (A.30) and the matching of solution (18) with solution (A.30) in $\mathrm{NF}^{\mathrm{s}}$ yields

$$
\theta(\epsilon)=\epsilon^{\beta} \quad \text { with } \beta=\frac{1}{1-\lambda_{1}},
$$


and

$$
v_{0}=\left(\frac{\sqrt{3} A_{\mathrm{s}}^{2 \lambda_{2}}}{\left(r^{+}-r^{-}\right)^{\lambda_{1}}} \int_{0}^{A_{\mathrm{s}}} a^{-\lambda_{1}}\left(A_{\mathrm{s}}^{2}-a^{2}\right)^{-\lambda_{2}-1} \mathrm{~d} a\right)^{\beta},
$$

$\lambda_{2}$ being given by Eq. (A.27) of Appendix A. The position of the maximum is given by the sum of the contribution in IL found by Eq. (A.33) from $x=0$ to $x_{i}$, where $\xi=1$ :

$$
x_{i} \simeq \frac{1}{r^{+}} \log \left(\frac{r^{+}-r^{-}}{v_{0}}\right),
$$

and the contribution in $\mathrm{NF}^{\mathrm{s}}$ and $\mathrm{TL}^{\mathrm{s}}$ found by Eq. (A.25) from $x_{i}$, where $A=\theta(\epsilon)$ to $x_{\mathrm{s}}$ :

$$
x_{\mathrm{s}}-x_{i} \simeq \frac{1}{k_{\mathrm{s}}^{-}}\left(2 \beta \log \epsilon+\log \mu_{1}\right),
$$

where $k_{\mathrm{s}}^{-}=-2 A_{\mathrm{s}}^{2} / \sqrt{3}$. From the condition $\mu_{1} \log \left(1 / \mu_{1}\right) \ll \epsilon \ll 1$, we obtain $\log (1 / \epsilon) \ll \log \left(1 / \mu_{1}\right)$. Therefore, when keeping only the dominant term in $x_{\mathrm{s}}$, we find

$$
x_{\mathrm{s}} \simeq \frac{1}{\left|k_{\mathrm{s}}^{-}\right|} \log \left(\frac{1}{\mu_{1}}\right) \text {. }
$$

Our analysis is, therefore, valid for $\epsilon \gg \mu_{1} \log \left(1 / \mu_{1}\right)$ and the dominant contribution to the position of the maximum $x_{\mathrm{s}}$ is given by (23), i.e., it scales as $\log \left(1 / \mu_{1}\right)$. This result differs from the one found for pulled global modes for which the location $x_{\mathrm{s}}$ of their maximum amplitude has been found to scale as $1 / \sqrt{\epsilon}$, as for the growth length of their homogeneous counterpart.

The dominant part $\log \left(1 / \mu_{1}\right)$ of the scaling law for $x_{\mathrm{S}}$ has been shown to exceed the growth length of homogeneous NG modes which scales as $\log (1 / \epsilon)$. This difference comes from the fact that a homogeneous NG mode never reaches a maximum but asymptotes it at $+\infty$. Its growth length is mainly given by the distance from the inlet necessary to grow out of the inner layer. In contrast, an NG mode not only grow out of the inner layer over the same distance, but also needs an extra length which is $\mathcal{O}\left[\log \left(1 / \mu_{1}\right)\right]$ to reach its maximum amplitude. Since our analysis is valid when both parameters $\epsilon$ and $\mu_{1}$ go to zero, with the condition $\epsilon \gg \mu_{1} \log \left(1 / \mu_{1}\right)$, the dominant contribution in the position of the maximum of the NG mode is constituted by this extra length.

\section{Numerical simulations in the oscillatory case and frequency selection problem}

In physical systems where the instability is oscillatory, the Ginzburg-Landau model (1) studied in the previous sections must be extended to the case where all coefficients are complex and vary with $x$. We will show numerically that:

1. the spatial structure of the pushed global modes found in the framework of the real equation (1) persists for the complex case where Eq. (1) is replaced by

$$
\frac{\partial A}{\partial t}+U_{0} \frac{\partial A}{\partial x}=\left(1+\mathrm{i} c_{1}\right) \frac{\partial^{2} A}{\partial x^{2}}+\mu A+\left(1+\mathrm{i} c_{3}\right)|A|^{2} A-\left(1-\mathrm{i} c_{5}\right)|A|^{4} A .
$$

In that case, we will comment on the frequency selection and on the stability of such global modes.

2. New states where the long time behavior is not time-periodic appear for particular values of the $c_{i}$ parameters. 
Although the results extend to cases where all coefficients vary, we will consider that only the bifurcation parameter $\mu$ and the advection velocity $U_{0}$ vary with $x$, while other coefficients including $c_{1}, c_{3}, c_{5}$ and those set to unity are kept constant. Numerical simulations of Eq. (24) have been performed with the initial condition $A(x, t=0)=0.8$ for $3<x<50$ and $A(x, t=0)=0$ otherwise. When the state $A=0$, referred to as the medium, is stable, this localized initial condition with finite amplitude triggers the evolution to an ultimate state, either oscillatory in the form of a pushed global mode with a well-defined frequency, or exhibiting a more disordered behavior. When the medium is unstable, however, the dynamics is triggered by an initial condition with infinitesimal amplitude. In any case, apart from this threshold effect, the final nonlinear saturated state of the system is not very sensitive to the initial condition, provided the latter is sufficiently localized.

\subsection{Pushed global modes for the complex Ginzburg-Landau model}

Fig. 6 shows the amplitude and real part of the pushed global mode obtained for the set of parameters close to the real case: $U_{0}=0.02, \mu(x)=(1+0.1 \mathrm{i})\left(-0.05-10^{-3} x\right), c_{1}=c_{3}=c_{5}=0$. The asymptotic state is a pushed global mode such as that described in the previous sections (Fig. 3(a)), with an upstream front stopped at the inlet and a bulk region where the saturated amplitude following the variation of parameters is limited downstream by a backward facing front. The position of this downstream front at $x_{\mathrm{d}}=141.7$ coincides with that marked by the small tick on the axis in Fig. 6(a) which is given by the condition $\operatorname{Re}\left(\mu\left(x_{\mathrm{d}}\right)\right)=\mu_{\mathrm{d}}\left(U_{0}\right)$, where $\mu_{\mathrm{d}}$ is given by Eq. (16). In this case, the saddle-node bifurcation where the local saturated wave stops to exist is located at $x_{\mathrm{SN}}=200$, where $\mu\left(x_{\mathrm{SN}}\right)=-\frac{1}{4}$. In Fig. 6(b), another pushed global mode is shown. It has been obtained asymptotically for long times for the parameters $U_{0}=0.6, \mu(x)=0.05-10^{-3} x, c_{1}=0.1, c_{3}=0.3, c_{5}=-0.1$. The departure from threshold $\epsilon=\mu_{0}-\mu_{A}=7.3 \times 10^{-3}$ is comparable to the inhomogeneity parameter $\mu_{1}=10^{-3}$. The position of the upstream front is, therefore, very close to the point marked by the tick at $x=7.32$ on the axis where $\mu(x)=\mu_{A}\left(U_{0}\right)$. In contrast with the pushed global modes in Fig. 6(a) where a decreasing front constituted the tail of the solution, the amplitude in Fig. 6(b) returns to zero due to the saddle-node bifurcation induced by the variation of the parameters. This case is the complex analogous of the mode in Fig. 3(b). The second tick at $x_{\mathrm{SN}}=300$ marks the position beyond which the saturated wave no longer exists since $\mu(x)<-\frac{1}{4}$. The difference in the way the tail
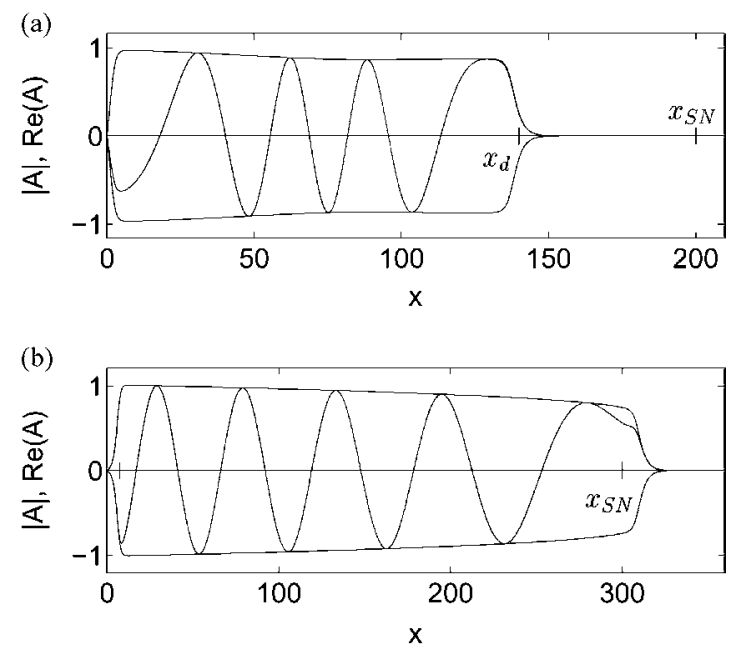

Fig. 6. (a) Envelope $|A|$ and real part $\operatorname{Re}(A)$ of pushed global modes with pushed front stopped by the boundary $x=0$ and sharp tail in the form of a decreasing front. (b) Same as in (a) but a saddle-node bifurcation induces the return to zero in the tail. 
of the global mode ends is subtle since the decrease to zero is abrupt in both cases, whether it is due to a backward facing front stopped at the location its speed vanishes as in Figs. 3(a) and 6(a) or it is due to the loss of existence of the saturated wave solution as in Figs. 3(b) and 6(b). Only the location of this abrupt decrease differentiates the two cases, the backward facing front being located closer to the origin than the location of the saddle-node bifurcation. Both locations are precisely computed from the theory and agree remarkably well with the numerical simulations.

When such a pushed global mode is obtained, it oscillates at a global frequency independent of the streamwise location $x$. This frequency is selected by the upstream front which acts as a wave maker for the entire medium. For example, the measured frequency of the pushed global mode presented in Fig. 6(b) is $\omega=0.12 \pm 0.01$ and should be compared to the theoretical value for the pushed front frequency $\omega^{\dagger}=0.115$ (obtained from Eq. (3.39c) in [32]). Since the departure from threshold is small, the agreement between the pushed front frequency and the pushed global mode frequency is here excellent. For comparison with these values, the frequency of the non-selected pulled front given by Eq. (4.8c) in [32] is $\omega^{*}=0.0089$. This value that would have been the one predicted by the linear criterion for front selection is totally in disagreement with the numerical results. For larger departures from threshold, the presence of the boundary slightly distorts the frequency of the pushed front. A phase space analysis similar to that presented in [8] in the case of pulled global modes would allow us to compute these distortions exactly, but we do not have attempted to use this technique here since the variation is second-order and the leading order result is already striking.

\subsection{Non-periodic modes and disordered behavior}

Due to the variation of the parameters, more complicated situations may arise: a pushed global mode is not systematically selected by the dynamics in Eq. (24). We will present two cases where a more disordered solution appears.

1. When the saturated amplitude wave that a pushed global mode follows adiabatically between the upstream and the downstream front becomes absolutely unstable, the global mode becomes unstable and a new state is made of the former pushed global mode with a front of secondary instability invading the tail. Such a scenario relying on an absolute secondary instability for the destabilization of a global mode has been shown to occur for homogeneous global modes when the saturated plane wave with the frequency selected by the pulled upstream front becomes absolutely unstable [8]. In the present case, no systematic study of the instability nature of the saturated plane waves with the frequency selected by the pushed upstream front will be made; this would constitute an extension of the results presented in [11] but would involve too many parameters to be exhaustive. The secondary instability induced by the variation of the parameters will, however, be shown on an ad hoc example. From numerical simulations with the parameters $U_{0}=0.6-0.1[1+\tanh (0.02 x-3)], \mu(x)=0.09-6 \times 10^{-4} x[1+\tanh (0.02 x-5)]$, $c_{1}=0.1, c_{3}=0.3$ and $c_{5}=-1$, Fig. 7(a) shows that the solution does not converge to an oscillating mode with a single frequency. Although the solution does not reach globally a time-periodic state, the upstream part of a pushed global mode, including the front blocked on the boundary and a part of the saturated tail, is obtained asymptotically for long times; the tail, however, cannot follow the slowly varying saturated wave at the frequency imposed by the upstream front since it becomes absolutely unstable and the global mode loses stability. The chaotic dynamics obtained in the tail constitutes a signature of the secondary bifurcation occurring in the system. The spatial structure of the solution then reflects the successive bifurcations which leave their signature at different positions.

2. More complicated situations may happen for large values of $c_{1}, c_{3}$ or $c_{5}$. In Fig. 7(b), it is shown that for the parameters $U_{0}=0.2, \mu(x)=0.032-8 \times 10^{-4} x, c_{1}=2, c_{3}=0.3$ and $c_{5}=0.4$, neither a global mode nor an oscillatory state is eventually obtained for long times. This may seem surprising since the criteria for the front selection problem predict the selection of a pulled front with velocity $v^{*}=0.8$ [32]. Here, the advection velocity 

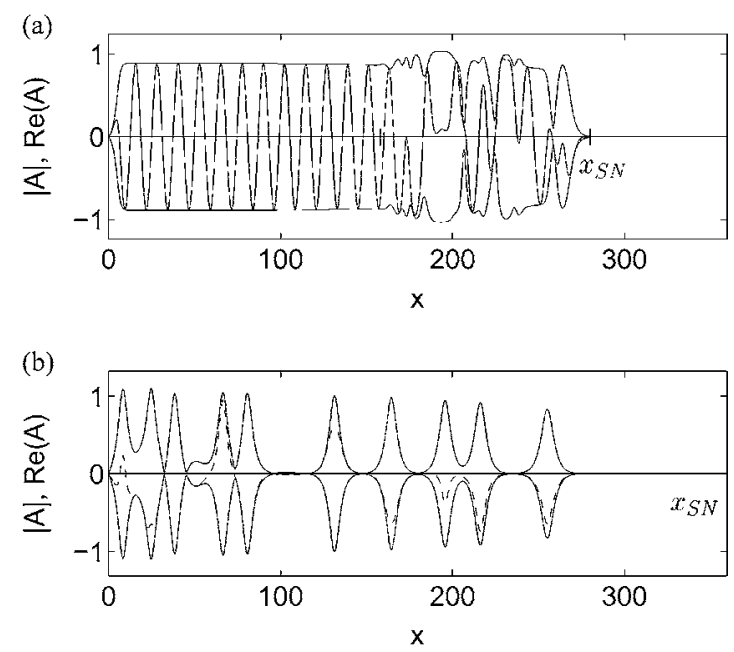

Fig. 7. (a) Envelope $|A|$ and real part $\operatorname{Re}(A)$ of pushed global modes with disordered behavior in the tail as the saturated traveling wave becomes unstable when $x$ varies. (b) Example of solution in parameters region where neither a pushed mode nor a pulled mode is selected. The real part of the amplitude is shown in dashed line.

is a fixed parameter and is smaller than $v^{*}$. Therefore, if the existence of an NG mode could be obtained from the front selection problem, a pulled front would be able to withstand the advection velocity, and a pulled global mode would be a possible asymptotic state.

In the present case, pulses are recurrently nucleated from the region of absolute instability bounded by the origin and the first tick on the axis, and then travel along the convective and stable region, beyond the second tick. Although no explanation is presently available for such a global mode, we recall that in an infinite medium without advection, van Saarloos and Hohenberg [32] predict the selection of stable pulses for a bifurcation parameter smaller than that for which the pushed front velocity vanishes. Here, the presence of the mean advection seems to shift the parameter region in which this pulse regime occurs and we, therefore, observe traveling pulses where a pulled global mode was expected. One may imagine that this is due to the instability of the rising front of the global mode being in that sense similar to the so-called core instability of Nozaki-Bekki holes or spiral solutions described in [53-55].

\section{Discussion of results and conclusion}

A complete description of self-synchronized structures in a semi-infinite open flow is now achieved with the identification of the new structure, called pushed global mode, derived from the model of the present paper. In the following, we briefly recall our results and the different open shear flows to which each nonlinear global mode pertains.

In the parallel flow case, the nonlinear global instability may be either of the Kolmogorov type, i.e., occurring when the basic state becomes absolutely unstable, or of the nonlinear type, i.e., occurring while the basic state is still convectively unstable. Whether the advection velocity is small or large distinguishes both situations; however, the nonlinear transition does not necessarily occur for small advection velocities. Nor is it restricted to subcritical systems. Addition of nonlinear advection terms in a supercritical model ("van der Pol-Duffing" system in Ref. [20]) indeed leads to a nonlinear transition for large advection velocities. In the case of a Kolmogorov transition, 
the growth length of homogeneous nonlinear global modes has been shown [20] to scale as $\epsilon^{-1 / 2}$, where $\epsilon$ is the departure from threshold. In the case of a nonlinear transition, the growth length of homogeneous nonlinear global modes scales as $\log (1 / \epsilon)$.

In the weakly non-parallel flow case, the nonlinear global instability may also be of both types. In the case of the Kolmogorov transition, pulled global modes are obtained when a sufficiently large domain of absolute instability in the medium triggers the global instability. The position of their maximum amplitude scales as $\epsilon^{-1 / 2}$. For the model of the present paper, these pulled modes are obtained for large advection velocities $\left(U_{0}>\sqrt{3}\right)$. They are also relevant to describe wakes when inhomogeneity is sufficiently weak as shown in [11]. In the case of the nonlinear transition, pushed global modes are obtained above the threshold of global instability, which is triggered even if no absolute instability region is present in the medium. The position of the maximum amplitude of the pushed global modes scales as $\log \left(1 / \mu_{1}\right)$ and, to our knowledge, this scaling has not been observed experimentally in a weakly non-parallel flow. These pushed global modes whose spatial structure has been identified on the real Ginzburg-Landau model (1) are also relevant to systems where the instability is oscillatory as shown on the complex Ginzburg-Landau model. In this case, the frequency is selected by the pushed front and differs strongly from the frequency predicted by the linear criterion for the front selection. More disordered behaviors are, however, not precluded.

A $\log (1 / \epsilon)$ scaling has been obtained in the parallel flow case for the growth length in the Kelvin-Helmholtz unstable interface in a Hele-Shaw cell by Gondret et al. [3]. The $\log \left(1 / \mu_{1}\right)$ scaling could be obtained in a similar experiment by addition of a weak inhomogeneity. We conclude this paper by emphasizing that, although the $\log \left(1 / \mu_{1}\right)$ scaling is not specific to subcritical systems, an experiment known to display a subcritical behavior (which could be achieved by adding a Poiseuille flow) may lead to the observation of the latter scaling. Görtler flow seems to us a good candidate where these scalings for the pushed global modes could be observed. In optical parametric oscillators [2], pushed global modes with the spatial structure of the present paper have been already identified; hence, the latter system is also well suited to test the scaling laws of the present paper.

\section{Appendix A. Detailed spatial structure of pushed global modes}

Since the spatial structure of the solution is similar in $\mathrm{NF}^{\mathrm{s}}$ and $\mathrm{NF}^{\mathrm{d}}$, and also in $\mathrm{TL}^{\mathrm{s}}$ and $\mathrm{TL}^{\mathrm{d}}$, we will use the following notations throughout the appendices to avoid the double description of the layers.

$$
\begin{aligned}
& \mu_{\mathrm{s}} \equiv \mu_{A}, \quad A_{\mathrm{s}} \equiv A_{2}\left(\mu_{A}\right), \\
& A_{\mathrm{d}} \equiv A_{2}\left(\mu_{\mathrm{d}}\right) .
\end{aligned}
$$

\section{A.1. Outer layer CNL}

In this layer, the amplitude varies weakly with respect to the slow variable $X=\mu_{1} x$. The change of variable $\mathcal{X}=\mu_{1} x-\mu_{0}$, where $\mu_{0}=\mu_{A}+\epsilon$ leads to seek a solution of

$$
\mathcal{X} A-A^{3}+A^{5}+\mu_{1} U_{0} A^{\prime}(\mathcal{X})=0
$$

where prime denotes differentiation with respect to the argument. Since $\mu_{0} \ll 1$ and the derivative $A^{\prime}(\mathcal{X})$ only appears at first-order in $\mu_{1}$, Eq. (A.3) is not an ordinary differential equation and its solution is obtained at each order without free parameter:

$$
A(\mathcal{X})=A_{0}(\mathcal{X})+\mu_{1} A_{1}(\mathcal{X})
$$


with

$$
A_{0}(\mathcal{X})=\left(\frac{1}{2}+\frac{1}{2} \sqrt{1-4 \mathcal{X}}\right)^{1 / 2}, \quad A_{1}(\mathcal{X})=\frac{U_{0}}{4 A_{0}^{3}(\mathcal{X})\left[1-2 A_{0}^{2}(\mathcal{X})\right]^{2}} .
$$

The position of CNL is, therefore, fixed in space and delimited by the upstream boundary $x_{\mathrm{s}}$, where $A(x)$ is maximum and the downstream boundary $x_{\mathrm{d}}$, where $\mu\left(x_{\mathrm{d}}\right)=\mu_{\mathrm{d}}\left(U_{0}\right)$. The solution (A.4) and (A.5) may be expanded near the CNL boundaries which correspond to the values $\mathcal{X}=-\mu\left(x_{i}\right)+\mu_{1}\left(x-x_{i}\right), i=\mathrm{s}$, d. Note that $\mu\left(x_{\mathrm{s}}\right)=$ $\mu_{A}+\epsilon-\mu_{1} x_{\mathrm{s}}$ whereas with $\mu\left(x_{\mathrm{d}}\right)=\mu_{\mathrm{d}}$. This leads to the following expansions, which are not totally symmetric due to the definition of the CNL boundaries:

$$
\begin{aligned}
\text { when } x \rightarrow x_{\mathrm{s}}: & A(\mathcal{X}) \simeq A_{0}\left(-\mu_{A}\right)+\left(\mu_{1} x-\epsilon\right) A_{0}^{\prime}\left(-\mu_{A}\right)+\mu_{1} A_{1}\left(-\mu_{A}\right), \\
\text { when } x \rightarrow x_{\mathrm{d}}: & A(\mathcal{X}) \simeq A_{0}\left(-\mu_{\mathrm{d}}\right)+\mu_{1}\left(x-x_{\mathrm{d}}\right) A_{0}^{\prime}\left(-\mu_{\mathrm{d}}\right)+\mu_{1} A_{1}\left(-\mu_{\mathrm{d}}\right) .
\end{aligned}
$$

\section{A.2. Transition layers $T L^{s}$ and $T L^{d}$}

In these two layers, the analysis is similar since we seek a solution of Eq. (12) linearized around one of the boundary $x_{i}$ of CNL, which represents also the center of the layer $\mathrm{TL}^{i}(i=\mathrm{s}, \mathrm{d})$, and where the amplitude is close to a finite value $A_{i}$ (see Eqs. (A.1) and (A.2)). We, therefore, introduce $A=A_{i}-\epsilon^{p_{i}} \phi_{i}(x)$ into Eq. (12) and expand the latter at first-order in $\epsilon^{p_{i}}$. We obtain the equation satisfied by $\phi_{i}(x)$ :

$$
\frac{\mathrm{d}^{2} \phi_{i}}{\mathrm{~d} x^{2}}-U_{0} \frac{\mathrm{d} \phi_{i}}{\mathrm{~d} x}+\tilde{\mu}_{i} \phi_{i}=\epsilon^{-p_{i}} A_{i} \psi_{i}(x)
$$

where the quantity $\psi_{i}(x)$ on the right-hand side reads as

$$
\psi_{\mathrm{s}}(x)=\left(\epsilon-\mu_{1} x\right), \quad \psi_{\mathrm{d}}(x)=-\mu_{1}\left(x-x_{\mathrm{d}}\right),
$$

and $\tilde{\mu}_{i}=\mu_{i}+3 A_{i}^{2}-5 A_{i}^{4}$ with the convention $\mu_{\mathrm{s}} \equiv \mu_{A}$. Using the notations

$$
k_{\mathrm{s}}^{-}=-\frac{1}{2}\left(U_{0}+\sqrt{3}\right), \quad k_{\mathrm{s}}^{+}=\frac{1}{2}\left(3 U_{0}+\sqrt{3}\right), \quad k_{\mathrm{d}}^{-}=\frac{1}{2}\left(3 U_{0}-\sqrt{3}\right), \quad k_{\mathrm{d}}^{+}=-\frac{1}{2}\left(U_{0}+\sqrt{3}\right),
$$

the general solution of Eq. (A.8) then reads as

$$
\phi_{i}(x)=c_{1 i} \mathrm{e}^{k_{i}^{+}\left(x-x_{i}\right)}+c_{2 i} \mathrm{e}^{k_{i}^{-}\left(x-x_{i}\right)}+\epsilon^{-p_{i}} \frac{A_{i}}{\tilde{\mu}_{i}} \psi_{i}(x)-\mu_{1} \epsilon^{-p_{i}} \frac{U_{0} A_{i}}{\tilde{\mu}_{i}^{2}},
$$

where the last non-exponential term represents a particular solution of Eq. (A.8) and $c_{1 i}, c_{2 i}$ are integration constants. In each transition layer $\mathrm{TL}^{\mathrm{s}}$ and $\mathrm{TL}^{\mathrm{d}}$, one boundary condition will be imposed by the matching with the solution in CNL when $x-x_{\mathrm{S}} \rightarrow+\infty$, and when $x-x_{\mathrm{d}} \rightarrow-\infty$. The outer solution (A.6) in CNL is linear with respect to $x$; then $\epsilon^{p_{\mathrm{s}}} \phi_{\mathrm{s}}(x)$ and $\epsilon^{p_{\mathrm{d}}} \phi_{\mathrm{d}}(x)$ must be also linear when $x-x_{\mathrm{s}} \rightarrow+\infty$ and $x-x_{\mathrm{d}} \rightarrow-\infty$, respectively. These two matching conditions imply $c_{1 \mathrm{~s}}=0$ and $c_{2 \mathrm{~d}}=0$. When $x-x_{\mathrm{s}} \rightarrow+\infty$, or $x-x_{\mathrm{d}} \rightarrow-\infty$, the inner solution (A.11) admits the expansion

$$
\epsilon^{p_{i}} \phi_{i}(x) \simeq \frac{A_{i}}{\tilde{\mu}_{i}} \psi_{i}(x)-\mu_{1} \epsilon^{-p_{i}} \frac{U_{0} A_{i}}{\tilde{\mu}_{i}^{2}} .
$$

Since $A_{0}\left(-\mu_{i}\right)=A_{i}, A_{i} / \tilde{\mu}_{i}=A_{0}^{\prime}\left(-\mu_{i}\right)$ and $U_{0} A_{i} / \tilde{\mu}_{i}^{2}=A_{1}\left(-\mu_{i}\right)$, expansion (A.12) with $i=\mathrm{s}[i=\mathrm{d}]$, represents exactly orders $\epsilon$ and $\mu_{1}$ of the solution (A.6) [the solution (A.7)], respectively. Hence, matching between $\mathrm{CNL}$ and $\mathrm{TL}^{i}$ is done. The quantities $c_{1 \mathrm{~d}}$ and $c_{2 \mathrm{~s}}$ must still be determined. 
The position $x_{\mathrm{S}}$ of the maximum amplitude $x_{\mathrm{s}}$ must satisfy $\mathrm{d} \phi_{\mathrm{s}} / \mathrm{d} x\left(x_{\mathrm{S}}\right)=0$. This condition determines the constant $c_{2 \mathrm{~s}}=\mu_{1} \epsilon^{-p_{\mathrm{s}}} A_{\mathrm{s}} / \tilde{\mu}_{\mathrm{s}} k_{\mathrm{s}}^{-}$. The quantity $x_{\mathrm{d}}$ is defined by $\mu\left(x_{\mathrm{d}}\right)=\mu_{\mathrm{d}}$ and hence, $A\left(x_{\mathrm{d}}\right)=A_{\mathrm{d}}$ yields the condition $\phi_{\mathrm{d}}\left(x_{\mathrm{d}}\right)=0$, which determines the constant $c_{1 \mathrm{~d}}=\mu_{1} \epsilon^{-p_{\mathrm{d}}} A_{\mathrm{d}} U_{0} / \tilde{\mu}_{\mathrm{d}}^{2}$. The complete solution (A.11) with $i=\mathrm{s}[i=\mathrm{d}]$ is known and in order to match it with the front solution in $\mathrm{NF}^{\mathrm{s}}\left[\mathrm{NF}^{\mathrm{d}}\right]$, we determine its asymptotic behavior when $x-x_{\mathrm{S}} \rightarrow-\infty\left[x-x_{\mathrm{d}} \rightarrow+\infty\right]$, respectively (given by the exponential terms at leading order). Since the matching of these parts of solution will be done in the phase space, we differentiate Eq. (A.11) and combine the result with Eq. (A.11) again to eliminate the exponential term which appears in the differentiated equation:

$$
\begin{aligned}
& \epsilon^{p_{\mathrm{s}}} \frac{\mathrm{d} \phi_{\mathrm{s}}}{\mathrm{d} x}=k_{\mathrm{s}}^{-} \epsilon^{p_{\mathrm{s}}} \phi_{\mathrm{s}}+\mu_{1} x_{\mathrm{s}} \frac{k_{\mathrm{s}}^{-} A_{\mathrm{s}}}{\tilde{\mu}_{\mathrm{s}}}-\epsilon \frac{k_{\mathrm{s}}^{-} A_{\mathrm{s}}}{\tilde{\mu}_{\mathrm{s}}}+\mu_{1} \frac{A_{\mathrm{s}}}{\tilde{\mu}_{\mathrm{s}}}\left(\frac{U_{0} k_{\mathrm{s}}^{-}}{\tilde{\mu}_{\mathrm{s}}}-1\right), \\
& \epsilon^{p_{\mathrm{d}}} \frac{\mathrm{d} \phi_{\mathrm{d}}}{\mathrm{d} x} \simeq k_{\mathrm{d}}^{+} \epsilon^{p_{\mathrm{d}}} \phi_{\mathrm{d}} .
\end{aligned}
$$

Eq. (A.13) is an exact relation and will be used as the asymptotic behavior of the solution (A.11) in the phase space as $x-x_{\mathrm{s}} \rightarrow-\infty$, whereas (A.14) is truncated at leading order which is sufficient to do the matching when $x-x_{\mathrm{d}} \rightarrow+\infty$.

Moreover, the maximum amplitude reads as $A\left(x_{\mathrm{s}}\right)=A_{\mathrm{s}}-\epsilon^{p_{\mathrm{s}}} \phi\left(x_{\mathrm{s}}\right)$ with

$$
\epsilon^{p_{\mathrm{s}}} \phi\left(x_{\mathrm{s}}\right)=-\mu_{1} \frac{A_{\mathrm{s}}}{\tilde{\mu}_{\mathrm{s}}} x_{\mathrm{s}}+\epsilon \frac{A_{\mathrm{s}}}{\tilde{\mu}_{\mathrm{s}}}+\mu_{1} \frac{A_{\mathrm{s}}}{\tilde{\mu}_{\mathrm{s}}}\left(\frac{1}{k_{\mathrm{s}}^{-}}-\frac{U_{0}}{\tilde{\mu}_{\mathrm{s}}}\right) .
$$

\section{A.3. Central nonlinear layer $N F^{s}$ and $N F^{d}$}

In the front layer $\mathrm{NF}^{\mathrm{S}}\left[\mathrm{NF}^{\mathrm{d}}\right]$ which possesses one boundary at $x_{\mathrm{s}}\left[x_{\mathrm{d}}\right]$, we consider that the solution grows [decreases] sufficiently fast so that $\mu(x)$ is approximately constant and equal to $\mu_{\mathrm{s}}\left[\mu_{\mathrm{d}}\right]$. In other words, the size of the two front layers is much smaller than $\mu_{1}^{-1}$. The solution in $\mathrm{NF}^{\mathrm{s}}\left[\mathrm{NF}^{\mathrm{d}}\right]$ is then nearly the same as in the homogeneous problem that has been already solved [20]. Indeed, at leading order, the solution in $\mathrm{NF}^{\mathrm{s}}$ represents a heteroclinic trajectory in the phase space $(A, \mathrm{~d} A / \mathrm{d} x)$, linking the origin to the point $\left(A_{\mathrm{s}}, \mathrm{d} A / \mathrm{d} x=0\right)$ for the constant parameter value $\mu=\mu_{A}$, whereas in $\mathrm{NF}^{\mathrm{d}}$, the solution represents a heteroclinic trajectory linking the point $\left(A_{\mathrm{d}}, \mathrm{d} A / \mathrm{d} x=0\right)$ to the origin. At the next order, small corrections have to be added, due to the small variation of $\mu(x)$ over the size of $\mathrm{NF}^{\mathrm{s}}\left[\mathrm{NF}^{\mathrm{d}}\right]$.

We, therefore, seek these solutions directly in the phase space in the form of Eq. (A.16):

$$
u(A)=\frac{\mathrm{d} A}{\mathrm{~d} x}=u_{0}(A)+\epsilon u_{1}(A)+\mu_{1} u_{2}(A) .
$$

At the lowest order, $u_{0}(A)$ satisfies

$$
u_{0} u_{0}^{\prime}-U_{0} u_{0}+\mu_{i} A+A^{3}-A^{5}=0,
$$

where $i=\mathrm{s}$ or $i=\mathrm{d}$ and a prime denotes differentiation with respect to $A$. For the value of the parameter $\mu_{i}$ previously defined and an advection velocity $U_{0}<1 / \sqrt{3}$, both solutions $u_{0}(A)$ in $\mathrm{NF}^{\mathrm{s}}$ and $\mathrm{NF}^{\mathrm{d}}$ are polynomial. When $1 / \sqrt{3}<U_{0}<\sqrt{3}, u_{0}(A)$ is still polynomial in $\mathrm{NF}^{\mathrm{s}}$ but not in $\mathrm{NF}^{\mathrm{d}}$. Since the trailing edge of the NG mode in $\mathrm{NF}^{\mathrm{d}}$ and $\mathrm{OL}$ is slave of its spatial structure upstream, we present here only the case $U_{0}<1 / \sqrt{3}$ without loss of generality. For each layer $\mathrm{NF}^{i}$, the solution at leading order then reads

$$
u_{0}(A)= \pm \frac{1}{\sqrt{3}} A\left(A_{i}^{2}-A^{2}\right)
$$


with the plus sign if $i=\mathrm{s}$ and the minus sign if $i=\mathrm{d}$. This solution may be again integrated:

$$
A=A_{i}\left(1+A_{0 i}^{-2} \mathrm{e}^{-\left( \pm A_{i}^{2} / \sqrt{3}\right) x}\right)^{-1 / 2},
$$

where $A_{0 i}$ is an integration constant.

When $x \rightarrow x_{i}$, the asymptotic behavior of the solution reads as

$$
A=A_{i}-\frac{A_{i}}{2 A_{0 i}^{2}} \mathrm{e}^{-\left( \pm 2 A_{i}^{2} / \sqrt{3}\right) x} .
$$

Since $k_{\mathrm{s}}^{-}=-2 A_{\mathrm{s}}^{2} / \sqrt{3}\left[k_{\mathrm{d}}^{+}=2 A_{\mathrm{d}}^{2} / \sqrt{3}\right]$, the solution (A.20) with the plus (minus) sign matches with the solution (A.11) in $\mathrm{TL}^{\mathrm{s}}\left[\mathrm{TL}^{\mathrm{d}}\right]$, respectively. This determines the constants

$$
A_{0 \mathrm{~s}}^{-2}=\frac{2 \mu_{1}}{\tilde{\mu}_{\mathrm{s}} k_{\mathrm{s}}^{-}} \mathrm{e}^{-k_{\mathrm{s}}^{-} x_{\mathrm{s}}} \quad \text { and } \quad A_{0 \mathrm{~d}}^{-2}=\frac{2 \mu_{1} U_{0}}{\tilde{\mu}_{\mathrm{d}}^{2}} \mathrm{e}^{-k_{\mathrm{d}}^{+} x_{\mathrm{d}}} .
$$

By reporting these values in Eq. (A.19), we find the asymptotic behavior of the solution in TL ${ }^{\mathrm{d}}$ when $x-x_{\mathrm{d}} \rightarrow+\infty$ :

$$
A \simeq A_{\mathrm{d}} \frac{\tilde{\mu}_{\mathrm{d}}}{\sqrt{2 U_{0} \mu_{1}}} \mathrm{e}^{-k_{\mathrm{d}}^{+}\left(x-x_{\mathrm{d}}\right) / 2} .
$$

In $\mathrm{NF}^{\mathrm{d}}$, this leading order is sufficient to do the matching with $\mathrm{OL}$ as will be seen below, but in $\mathrm{NF}^{\mathrm{s}}$, we have to compute the correction to the leading order. This next order to be taken into account may be either $\epsilon$ or $\mu_{1} x$; we assume that they are separated; $u_{1}(A)$ and $u_{2}(x, A)$ satisfy

$$
\begin{aligned}
& u_{0} u_{1}^{\prime}+\left(u_{0}^{\prime}-U_{0}\right) u_{1}=-A, \\
& u_{0} u_{2}^{\prime}+\left(u_{0}^{\prime}-U_{0}\right) u_{2}=x A,
\end{aligned}
$$

where $x$ in (A.24) is at first-order the function of $A$ defined by Eq. (A.19), with the corresponding constant $A_{0 \mathrm{~s}}$, which reads

$$
x(A)=x_{\mathrm{s}}-\frac{1}{k_{\mathrm{s}}^{-}}\left[\log \left(\frac{A^{2}}{A_{\mathrm{s}}^{2}-A^{2}}\right)-\log \left(\frac{\tilde{\mu}_{\mathrm{s}} k_{\mathrm{s}}^{-}}{2 \mu_{1}}\right)\right] .
$$

Since $u_{0}(A)$ is a polynomial solution, $u_{1}$ and $u_{2}$ can be obtained analytically by introduction of Eq. (A.18) in Eqs. (A.23) and (A.24) and of Eq. (A.25) in Eq. (A.24). By integration of the resulting equations, we obtain

$$
u_{1}(A)=-\sqrt{3} A^{\lambda_{1}}\left(A_{\mathrm{s}}^{2}-A^{2}\right)^{\lambda_{2}} \int_{A_{\mathrm{s}}}^{A} a^{-\lambda_{1}}\left(A_{\mathrm{s}}^{2}-a^{2}\right)^{-\lambda_{2}-1} \mathrm{~d} a,
$$

where

$$
\lambda_{1}=\frac{3 U_{0}-\sqrt{3}}{U_{0}+\sqrt{3}}, \quad \lambda_{2}=-\frac{3 U_{0}+\sqrt{3}}{U_{0}+\sqrt{3}},
$$

and

$$
u_{2}(A)=\sqrt{3} A^{\lambda_{1}}\left(A_{\mathrm{s}}^{2}-A^{2}\right)^{\lambda_{2}} \int_{A_{\mathrm{s}}}^{A} a^{-\lambda_{1}}\left(A_{\mathrm{s}}^{2}-a^{2}\right)^{-\lambda_{2}-1} x(a) \mathrm{d} a,
$$

where $x(a)$ is the function of $a$ defined by Eq. (A.25). 
When $A \rightarrow A_{\mathrm{s}}$, the solution (A.16) may be expanded as

$$
u_{0}(A)+\epsilon u_{1}(A)+\mu_{1} u_{2}(A) \simeq-\frac{2 A_{\mathrm{s}}^{2}}{\sqrt{3}}\left(A_{\mathrm{s}}-A\right)-\epsilon \frac{\sqrt{3}}{2 A_{\mathrm{s}} \lambda_{2}}+\mu_{1} \frac{\sqrt{3}}{2 A_{\mathrm{s}} \lambda_{2}}\left(x-\frac{\sqrt{3}}{2 A_{\mathrm{s}}^{2} \lambda_{2}}\right),
$$

which is identical to expansion (A.13) since we verify that $k_{\mathrm{s}}^{-}=-2 A_{\mathrm{s}}^{2} / \sqrt{3}, k_{\mathrm{s}}^{-} A_{\mathrm{s}} / \tilde{\mu}_{\mathrm{s}}=\sqrt{3} / 2 A_{\mathrm{s}} \lambda_{2}$ and $\left(A_{\mathrm{s}} / \tilde{\mu}_{\mathrm{s}}\right)\left(U_{0} k_{\mathrm{s}}^{-} / \mu_{\mathrm{s}}-1\right)=-3 / 4 A_{\mathrm{s}}^{3} \lambda_{2}^{2}$. The solutions in $\mathrm{TL}^{\mathrm{s}}$ and $\mathrm{NF}^{\mathrm{s}}$ are now matched. In other words, the matching succeeds whatever the size of the transition layer $\mathrm{TL}^{\mathrm{S}}$. When $A \rightarrow 0$, the solution $u(A)$ defined by Eqs. (A.16), (A.18), (A.26) and (A.28) admits the expansion

$$
\begin{aligned}
& u_{0}(A)+\epsilon u_{1}(A)+\mu_{1} u_{2}(A) \\
& \simeq \frac{A_{\mathrm{s}}^{2}}{\sqrt{3}} A+\sqrt{3} A^{\lambda_{1}} A_{\mathrm{s}}^{2 \lambda_{2}}\left\{B\left[\epsilon-\mu_{1}\left(x_{\mathrm{s}}-\frac{1}{k_{\mathrm{s}}^{-}} \log \mu_{1}\right)-\frac{\mu_{1}}{k_{\mathrm{s}}^{-}} \log \frac{\tilde{\mu}_{\mathrm{s}} k_{\mathrm{s}}^{-}}{2}\right]+\mu_{1} \frac{\sqrt{3}}{A_{\mathrm{s}}^{2}} \Gamma\right\},
\end{aligned}
$$

where

$$
B=\int_{0}^{A_{\mathrm{s}}} a^{-\lambda_{1}}\left(A_{\mathrm{s}}^{2}-a^{2}\right)^{-\lambda_{2}-1} \mathrm{~d} a, \quad \Gamma=\int_{0}^{A_{\mathrm{s}}} a^{-\lambda_{1}}\left(A_{\mathrm{s}}^{2}-a^{2}\right)^{-\lambda_{2}-1} \log \frac{a}{\sqrt{A_{\mathrm{s}}^{2}-a^{2}}} \mathrm{~d} a .
$$

\section{A.4. Transition layer IL}

In this layer, the amplitude is small since $A$ vanishes at $x=0$. Denoting $\xi$ the inner variable which is connected to the amplitude by the relation $A=\theta(\epsilon) \xi$ (where $\theta(\epsilon)$ denotes the size in amplitude of the inner layer and will be precised by the matching), $\xi$ satisfies the linearized equation (12) around $A=0$ :

$$
\xi^{\prime \prime}-U_{0} \xi^{\prime}+\mu(x) \xi=0 .
$$

Since $\xi(x)$ must vanish at the origin, the solution of Eq. (A.31) may be written with one undetermined integration constant $v_{0}$, and using Airy functions [52]

$$
\xi(x)=\frac{v_{0} \pi}{\mu_{1}^{1 / 3}} \mathrm{e}^{U_{0} x / 2}\left\{a \operatorname{Bi}\left[\left(x-x_{K}\right) \mu_{1}^{1 / 3}\right]-b \operatorname{Ai}\left[\left(x-x_{K}\right) \mu_{1}^{1 / 3}\right]\right\},
$$

where $x_{K}=\left(\mu_{A}-\frac{1}{4} U_{0}^{2}+\epsilon\right) / \mu_{1}$ is the position at which $\mu(x)$ reaches $\frac{1}{4} U_{0}^{2}\left(x_{K}\right.$ is negative since $\left.\mu_{A}\left(U_{0}\right)<\frac{1}{4} U_{0}^{2}\right)$, $a=\operatorname{Ai}\left(-x_{K} \mu_{1}^{1 / 3}\right)$ and $b=\operatorname{Bi}\left(-x_{K} \mu_{1}^{1 / 3}\right)$. The slope $v_{0}$ at the origin of the inner solution (A.32) will be fixed by the matching. Since $\mu_{1} \ll 1$, we obtain $-x_{K} \mu_{1}^{1 / 3} \gg 1$. Hence, the Ai and Bi functions may be replaced by their asymptotic expansions at infinity. The same inner solution than for homogeneous NG mode [20] is found and read as

$$
\xi(x)=\frac{v_{0}}{r^{+}-r^{-}}\left(\mathrm{e}^{r^{+} x}-\mathrm{e}^{r^{-} x}\right)
$$

where $r^{+}=\frac{1}{4}\left(U_{0}+\sqrt{3}\right)$ and $r^{-}=\frac{1}{4}\left(3 U_{0}-\sqrt{3}\right)$.

\section{A.5. outer layer $O L$}

In the outer layer OL, $A(x)$ is small since it must vanish at infinity. Therefore, $A(x)$ is solution of the linear equation (A.31) and reads

$$
A(x)=g\left(\mu_{1}\right) \mathrm{e}^{\left(U_{0} / 2\right) x} \operatorname{Ai}\left[\left(x-x_{K}\right) \mu_{1}^{1 / 3}\right],
$$


where $g\left(\mu_{1}\right)$ is an integration constant (the coefficient of Bi must be zero in order to cancel the growing part of the general solution).

In order match the solutions in $\mathrm{OL}$ and $\mathrm{NF}^{\mathrm{d}}$, let us expand (A.34) when $x \rightarrow x_{\mathrm{d}}$. Since $x-x_{K}=x_{\mathrm{d}}-x_{K}+x-x_{\mathrm{d}}$, and $x_{\mathrm{d}}-x_{K} \sim\left(U_{0}+\sqrt{3}\right)^{2} / 16 \mu_{1}, x_{\mathrm{d}} \sim\left(\mu_{A}-\mu_{\mathrm{d}}\right) / \mu_{1}=\sqrt{3} U_{0} / 4 \mu_{1}$, we may use the asymptotic behavior of Ai at infinity [52] to find

$$
A(x) \simeq g\left(\mu_{1}\right) \mu_{1}^{1 / 6} \frac{\mathrm{e}^{\left[12 \sqrt{3} U_{0}^{2}-\left(U_{0}+\sqrt{3}\right)^{3}\right] / 96 \mu_{1}}}{\left[\pi\left(U_{0}+\sqrt{3}\right)\right]^{1 / 2}} \mathrm{e}^{-k_{\mathrm{d}}^{+}\left(x-x_{\mathrm{d}}\right) / 2},
$$

where we have used $-\frac{1}{2} k_{\mathrm{d}}^{+}=\frac{1}{2} U_{0}-\left[\mu_{1}\left(x_{\mathrm{d}}-x_{K}\right)\right]^{1 / 2}$. Eq. (A.35) must be identified with Eq. (A.22) and therefore

$$
g\left(\mu_{1}\right)=\frac{3}{16}\left(\frac{3 \pi}{2}\right)^{1 / 2} \mu_{1}^{-2 / 3} \mathrm{e}^{\left[\left(U_{0}+\sqrt{3}\right)^{3}-12 \sqrt{3} U_{0}^{2}\right] / 96 \mu_{1}}\left(1+\frac{\sqrt{3}}{U_{0}}\right)^{1 / 2}\left(U_{0}-\frac{1}{\sqrt{3}}\right)\left(U_{0}-\sqrt{3}\right)^{2} .
$$

\section{References}

[1] P. Huerre, P.A. Monkewitz, Local and global instabilities in spatially developing flows, Annu. Rev. Fluid Mech. 22 (1990) $473-537$.

[2] M. Taki, N. Ouarzazi, H. Ward, P. Glorieux, Nonlinear front propagation in optical parametric oscillators, J. Opt. Soc. Am. B 17 (2000) 997.

[3] P. Gondret, P. Ern, L. Meignin, M. Rabaud, Experimental evidence of a nonlinear transition from convective to absolute instability, Phys. Rev. Lett. 82 (1999) 1442-1445.

[4] H.W. Müller, M. Lücke, M. Kamps, Transversal convection patterns in horizontal shear flow, Phys. Rev. A 45 (1992) $3714-3726$.

[5] H.W. Müller, M. Lücke, M. Kamps, Convective patterns in horizontal flow, Europhys. Lett. 10 (1989) 451-456.

[6] P. Büchel, M. Lücke, D. Roth, R. Schmitz, Pattern selection in the absolutely unstable regime as a nonlinear eigenvalue problem: Taylor vortices in axial flow, Phys. Rev. E 53 (1996) 4764-4777.

[7] A. Couairon, J.-M. Chomaz, Pattern selection in the presence of a cross flow, Phys. Rev. Lett. 79 (1997) 2666-2669.

[8] A. Couairon, J.-M. Chomaz, Primary and secondary nonlinear global instability, Physica D 132 (1999) 428-456.

[9] S.M. Tobias, M.R.E. Proctor, E. Knobloch, Convective and absolute instabilities of fluid flows in finite geometry, Physica D 113 (1998) $43-72$.

[10] S.M. Tobias, M.R.E. Proctor, E. Knobloch, The role of absolute instability in the solar dynamo, Astron. Astrophys. 318 (1997) L55-L58.

[11] A. Couairon, J.-M. Chomaz, Fully nonlinear global modes in slowly varying flows, Phys. Fluid 11 (1999) 3688-3703.

[12] B. Pier, P. Huerre, J.-M. Chomaz, A. Couairon, Steep nonlinear global modes in spatially developing media, Phys. Fluid 10 (1998) 2433-2435.

[13] B. Pier, P. Huerre, J.-M. Chomaz, Physica D 148 (2001) 49.

[14] A.P. Bassom, K.M. Kuzanyan, A.M. Soward, A nonlinear dynamo wave riding on a spatially varying background, Proc. R. Soc. London A 455 (1999) 1443-1481.

[15] A.M. Soward, Thin aspect ratio $\alpha \omega$-dynamos in galactic discs and stellar shells, in: A. Ferriz-Mas, M. Núñez Jiménez (Eds.), Advances in Nonlinear Dynamos, The Fluid Mechanics of Astrophysics and Geophysics, in press.

[16] R.J. Briggs, Electron-Stream Interaction with Plasmas, MIT Press, Cambridge, MA, 1964.

[17] A. Bers, Space time evolution of plasma instabilities — absolute and convective, in: M.N. Rosenbluth, R.Z. Sagdeev (Eds.), Handbook of Plasma Physics, North-Holland, Amsterdam, 1983, pp. 451-517.

[18] E.M. Lifshitz, L.P. Pitaevskii, Physical Kinetics, Pergamon Press, London, 1981.

[19] A. Couairon, J.-M. Chomaz, Global instability in nonlinear systems, Phys. Rev. Lett. 77 (1996) 4015-4018.

[20] A. Couairon, J.-M. Chomaz, Absolute and convective instabilities, front velocities and global modes in nonlinear systems, Physica D 108 (1997) 236-276.

[21] G. Ahlers, D.S. Cannel, Vortex-front propagation in rotating Couette-Taylor flow, Phys. Rev. Lett. 50 (1983) 1583.

[22] J. Fineberg, V. Steinberg, Vortex-front propagation in Rayleigh-Bénard convection, Phys. Rev. Lett. 58 (1987) 1332.

[23] A. Hanna, A. Saul, K. Showalter, Detailed studies of propagating fronts in the iodate oxidation of arsenous acid, J. Am. Chem. Soc. 104 (1982) 3838 .

[24] A. Kolmogorov, I. Petrovsky, N. Piskunov, Investigation of a diffusion equation connected to the growth of materials, and application to a problem in biology, Bull. Univ. Moscow Ser. Int. Sec. A 1 (1937) 1.

[25] A.C. Newell, T. Passot, J. Lega, Order parameter equations for patterns, Annu. Rev. Fluid Mech. 25 (1993) 399.

[26] D.G. Aronson, H.F. Weinberger, Multidimensional nonlinear diffusion arising in population genetics, Adv. Math. 30 (1978) 33.

[27] M. Bramson, Mem. Am. Math. Soc. 285 (1983) 1. 
[28] W. van Saarloos, Dynamical velocity selection: marginal stability, Phys. Rev. Lett. 58 (1987) 2571.

[29] W. van Saarloos, Front propagation into unstable states: marginal stability as a dynamical mechanism for velocity selection, Phys. Rev. A 37 (1988) 211.

[30] W. van Saarloos, Front propagation into unstable states. II. Linear versus nonlinear marginal stability and rate of convergence, Phys. Rev. A 39 (1989) 6367.

[31] W. van Saarloos, P.C. Hohenberg, Pulses and fronts in the complex Ginzburg-Landau equation near a subcritical bifurcation, Phys. Rev. Lett. 64 (1990) 749.

[32] W. van Saarloos, P.C. Hohenberg, Fronts, pulses, sources and sinks in generalized complex Ginzburg-Landau equations, Physica D 56 (1992) 303.

[33] E. Ben-Jacob, H.R. Brand, G. Dee, L. Kramer, J.S. Langer, Pattern propagation in nonlinear dissipative systems, Physica D 14 (1985) 348.

[34] G. Dee, Dynamical properties of propagating front solutions of the amplitude equations, Physica D 15 (1985) 295.

[35] G. Dee, Propagation into an unstable state, J. Statist. Phys. 39 (1985) 705.

[36] G. Dee, J.S. Langer, Phys. Rev. Lett. 50 (1990) 749.

[37] J.A. Powell, A.C. Newell, C.K.R.T. Jones, Competition between generic and nongeneric fronts in envelope equations, Phys. Rev. A 44 (1991) 3636.

[38] G.C. Paquette, Y. Oono, Structural stability and selection of propagating fronts in semilinear parabolic partial differential equations, Phys. Rev. E 49 (1994) 2368.

[39] R.D. Benguria, M.C. Depassier, Speed of fronts of the reaction-diffusion equation, Phys. Rev. Lett. 77 (1996) 1171.

[40] E. Brunet, B. Derrida, Shift in the velocity of a front due to a cutoff, Phys. Rev. E 56 (1997) 2597.

[41] U. Ebert, W. van Saarloos, Universal algebraic relaxation of fronts propagating into an unstable state and implications for moving boundary approximations, Phys. Rev. Lett. 80 (1998) 1650.

[42] J.-M. Chomaz, A. Couairon, Propagating pattern selection and causality reconsidered, Phys. Rev. Lett. 84 (2000) $1910-1914$.

[43] B.J.A. Zielinska, J.E. Wesfreid, On the spatial structure of global modes in wake flow, Phys. Fluid 7 (1995) 1418-1424.

[44] S. Goujon-Durand, P. Jenffer, J.E. Wesfreid, Downstream evolution of the Bénard-von Kármán instability, Phys. Rev. E 50 (1994) 308.

[45] H. Ward, M.N. Ouarzazi, M. Taki, P. Glorieux, Transverse dynamics of optical parametric oscillators in presence of walk-off, Eur. Phys. J. D 3 (1998) 275-288.

[46] H. Ward, M.N. Ouarzazi, M. Taki, P. Glorieux, Influence of walk-off on pattern formations in non-degenerate optical parametric oscillators, Phys. Rev. E 63 (2001) 016604-6.

[47] N. Meunier, M.R.E. Proctor, D. Sokoloff, A.M. Soward, S.M. Tobias, Asymptotic properties of a nonlinear $\alpha \omega$-dynamo wave: period, amplitude and latitude dependence, Geophys. Astrophys. Fluid Dyn. 86 (1997) 249-285.

[48] J.-M. Chomaz, P. Huerre, L.G. Redekopp, Bifurcations to local and global modes in spatially developing flows, Phys. Rev. Lett. 60 (1988) 25-28.

[49] J.-M. Chomaz, A. Couairon, Against the wind, Phys. Fluid 11 (1999) 2977-2983.

[50] J.-M. Chomaz, Absolute and convective instabilities in nonlinear systems, Phys. Rev. Lett. 69 (1992) 1931-1934.

[51] C.M. Bender, S.A. Orszag, Advanced Mathematical Methods for Scientists and Engineers, McGraw-Hill, New York, 1978.

[52] M. Abramowitz, I.A. Stegun, Handbook of Mathematical Functions, Dover, New York, 1972.

[53] H. Chaté, P. Manneville, Stability of the Bekki-Nozaki hole solutions to the one-dimensional complex Ginzburg-Landau equation, Phys. Lett. A 171 (1992) 183-188.

[54] I. Aranson, L. Kramer, A. Weber, Core instability and spatiotemporal intermittency of spiral waves in oscillatory media, Phys. Rev. Lett. 72 (1994) 2316.

[55] A. Weber, L. Kramer, I.S. Aranson, L. Aranson, Stability limits of spirals and traveling waves in nonequilibrium media, Phys. Rev. A 46 (1992) R2992. 